Research Paper

\title{
The impact of the public budget deficit in promoting the development of the financial sector Gulf Cooperation Council countries as a model
}

Journal of

\section{TANMIYAT AL- RAFIDAIN}

\section{(TANRA)}

A scientific, quarterly, international, open access, and peer-reviewed journal

Vol. 40, No. 130

June 2021

(C) University of Mosul | College of Administration and Economics, Mosul, Iraq.

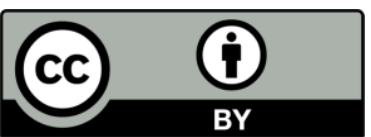

TANRA retains the copyright of published articles, which is released under a "Creative Commons Attribution License for CC-BY-4.0" enabling the unrestricted use, distribution, and reproduction of an article in any medium, provided that the original work is properly cited.

Citation: AL-Dabbgh, Nawar, AL-Iragi , Bashar A (2021). "The impact of the public budget deficit in promoting the development of the financial sector-Gulf Cooperation Council countries as a model". TANMIYAT AL-RAFIDAIN, 40 (130), 51 -76,

https://doi.org/

10.33899/tanra.2021.168685

P-ISSN: 1609-591X

e-ISSN: 2664-276X

tanmiyat.mosuljournals.com

\section{Nawar K. AL-Dabbagh ${ }^{1}$ Bashar A. AL-Iragi ${ }^{2}$}

University of Mosul, College of Business and Economics, Department of Banking and Financial Sciences

Corresponding author: Nawar K. AL-Dabbagh, University of Mosul, College of Business and Economics, Department of Banking and Financial Sciences

Nawarkanaan2019@gmail.com

DOI: https://doi.org/ 10.33899/tanra.2021.168685

Article History: Received: 12/8/2020; Revised: 31/8/2020; Accepted: 16/9/2020; Published: 1/6/2021.

\section{Abstract}

The research aims to provide a deep and comprehensive picture of the theoretical frameworks and empirical studies that dealt with the analysis and interpretation of the mechanism and channels of the impact that can be exercised by high levels of public budget deficit in strengthening and developing the financial sector, in addition to foreseeing an objective quantitative model that can diagnose the nature, size, and direction of this impact. And the interpretation of its transmission mechanism and channels, by adopting the methodology of aggregate regression models (PRM), static effect (FEM), and random effect (REM) that are based on the balanced longitudinal data (Balanced Panel Data) for the Gulf Cooperation Council countries for the period (2005-2017). The results of the assessment revealed the contribution of the public budget deficit variable in its negative impact on the financial sector development indicators, $(B C)$ which expresses the bank credit granted to the private sector as a percentage of GDP, and (TD) which refers to the bank credit granted as a percentage Of total bank deposits, while its negative impact was shared in the two financial sector development indicators, (SMC), which expresses the ratio of market value to the gross domestic product, and (SMT), which reflects the stock market turnover and the economic growth variable (GRO) also influenced Negative in both (BC) and (TD).

\section{Keywords}

Financial Sector, Financial Development, Public Budget Deficit, Economic Growth, Inflation, Balanced Panel Data Methodology. 


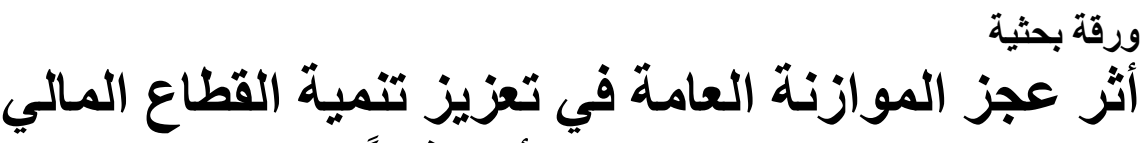

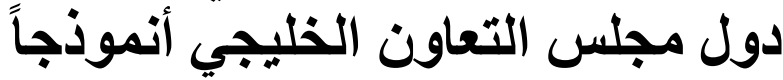

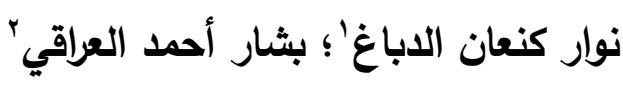

\begin{abstract}
جامعة الموصل، كلية الإدارة والاقتصاد، قسم العلوم المالية والمصرفية
\end{abstract}
المؤلف المراسل: نوار كنعان الدباغ، جامعة الموصل، كلية الإدارة والاقتصاد، قسم العلوم المالية والمصرفية

DOI: https://doi.org/ 10.33899/tanra.2021.168685

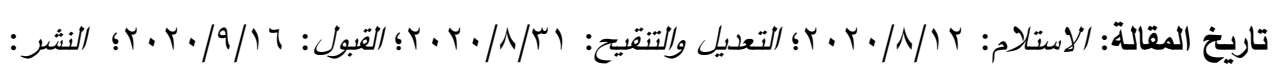

$$
\cdot r \cdot r / T / 1
$$

|لمستخلص

يحدف البحث إلى تقديم صورة عديقة وشاملة عن الأطر النظرية والدراسات التجريبية التي تناولت

تحليل وتغسير آلية وقنوات انتقال التأثير الذي بيكن أن بيارسه ارتغاع مستويات عجز الدوازنة العامة في

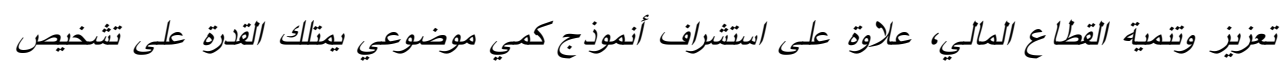
طبيعة التأثير وحجمه واتجاهه وتضسير آلية وقنوات انتقاله، من خلال اعتماد منهجية نداذج الانحدرار التجميعي (PRM) والأثر الثابت (PEM) والأثر العشوائسي (REM) التهي تستند إلى البيانات المزدوجة

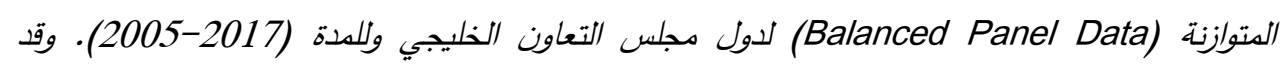

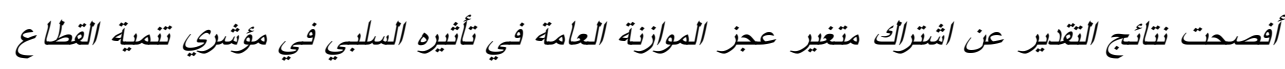
المالي ، (BC) الذي يعبر عن الائتمان المصرفي المهنوح للقطاع الخاص كنسبة من الناتج المحلي الإجمالي، و(TD) الذي يثير إلى الائتمان المصرفي المدنوح كنسبة من إجمالي الودائع المصرفية، في الدي حين اشترك بتأثيره السلبي في مؤشري تنمية القطاع المالي، (SMC) الذي يعبر عن نسبة القيهة السوقية

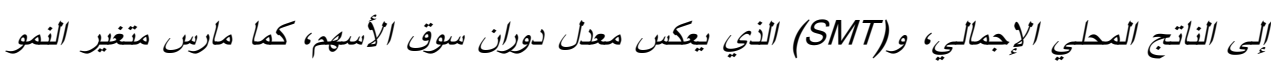

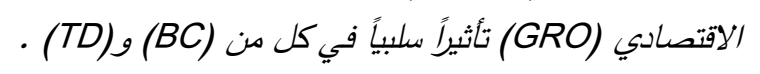

الكلمات المفتاحية

القطاع المالي، التنمية المالية، عجز الموازنة العامة، النمو الاقتصادي، التضخم، منهجية البيانات المزدوجة المتوازنة (Blanced panel Data)

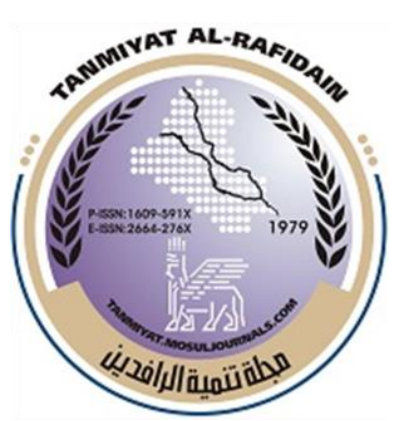

\section{هجلة}

\section{تنمية الرافدين}

(TANRA) دولية، مفتوحة الوصول، محكمة.

$$
\begin{aligned}
& \text { المجلد (•؛))، العدد (•r (1)، }
\end{aligned}
$$

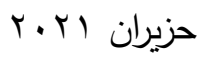

(C) جامعة الموصل | (c) كلية الإدارة والاقتصاد، الموصل، العراق.

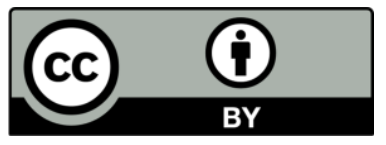

تحتفظ (TANRA) بحقوق الطبع والنشر للمقالات

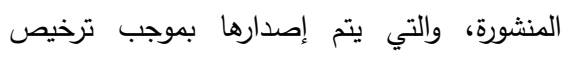

\lrcorner (Creative Commons Attribution) (CC-BY-4.0)

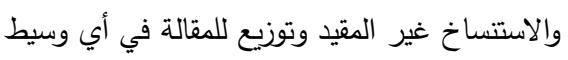
نقل، بشرط اقتباس العمل الأصلي بشكل صحيح.

الاقتباس: الدباغ، نوار ، العراقي ، بشار

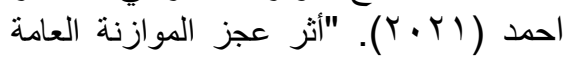

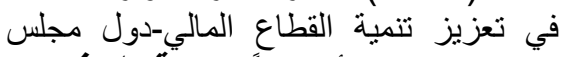

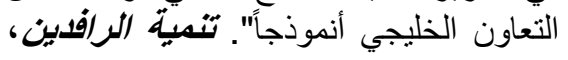

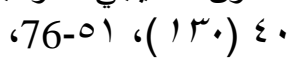

https://doi.org/

10.33899/tanra.2021.168685

P-ISSN: 1609-591X

e-ISSN: 2664-276X

tanmiyat.mosuljournals.com 
يؤدي القطاع المالي المتطور والكفوء وبحسب ما تجمع عليه معظم الادبيات المالية والاقتصادية'، ومن خلال قدرته على تعبئة المدخرات وإعادة توجيهها من الوحدات الاقتصادية ذات الفائض المالي نحو الوحدات ذات العجز المالي بما يضمن التخصيص الأمثل للموارد، وإدارة وتوزيع المخاطر ، وتيسير قنوات الوصول لتسوية المدفوعات وتقليل حالات اللاتماثل المعلوماتي، دوراً بالغ الأهمية في تعزيز النمو

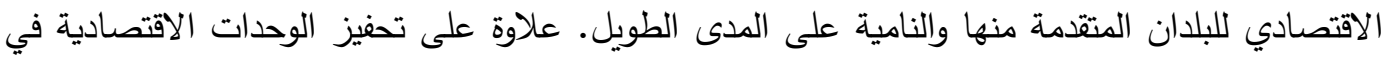
كافة القطاعات، والدفع بها نحو تحقيق مستويات عالية ومرغوبة من التطور والتتمية. عبر الأداء الكفوء والمتميز للمؤسسات المالية وتوفيرها لقنوات الوصول إلى أدوات وخدمات مالية متتوعة بسهولة وبتكلفة متدنية، كما يمكن للقطاع المالي، وفي ظل ظاهرة عدم اكتمال سوق رأس المال Capital Market)

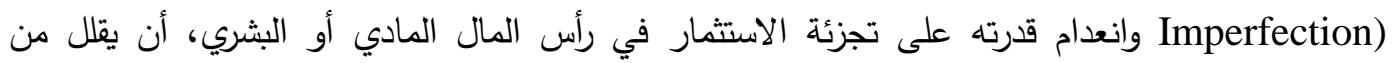
مستويات الفقر والتفاوت وعدم المساواة في توزيع الاخل بين أفراد المجتمع من خلال تخفيف قيود الائتمان الممنوح لذوي الاخول المنخفضة. وفي ضوء ما تقدم فان القطاع المالي يمثل حجر الزاوية للتطور

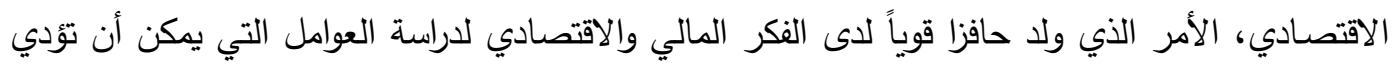
دوراً مهما وفاعلاً في دفع القطاع المالي نحو التتمية والتطور ، والوصول إلى أطر نظرية وتجريبية قادرة

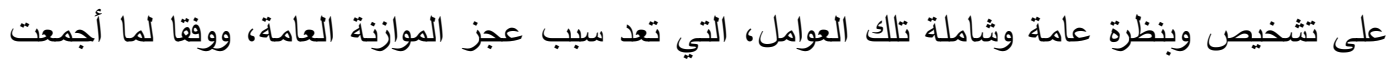

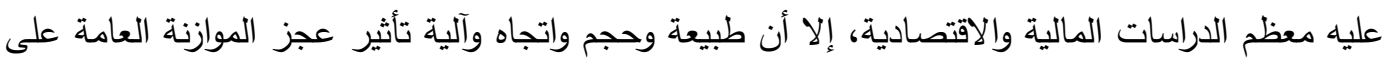

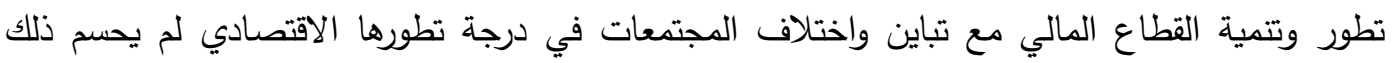
بصورة مطلقة، ما ولد حاجة متزايدة وملحة للبحث والتحليل.

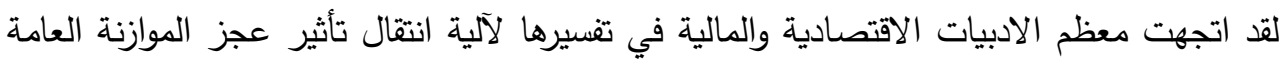
والناتج عن تجاوز الانفاق الحكومي مستويات إيراداتها والمغطى من خلال الاقتراض المباشر من الجهاز

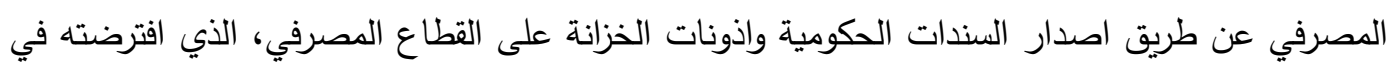
غالبيتها سالباً، عبر قناتين تتمثل الأولى باستباق أو سحب المدخرات المحلية من أمام القطاع المصرفي، في حين تتمثل الثانية باحتفاظ الجهاز المصرفي بجزء من تلك الاصدارات الحكومية، وفي كلا الحالتين

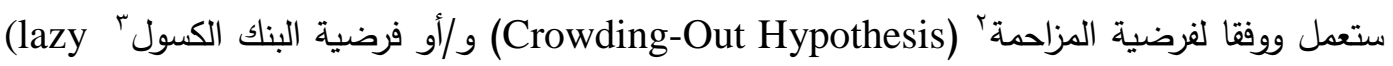

1. Christopoulos \& Tsionas (2004),King \& Levine (1993), Levine, Loayza \& Beck (2000), Calderon \& Liu (2003), Bayar, Gündüz \& Sezgin (2019)

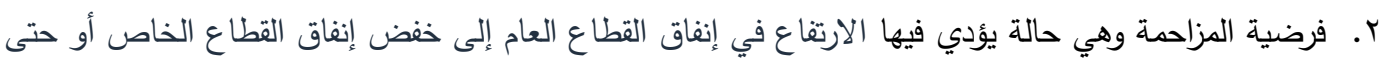

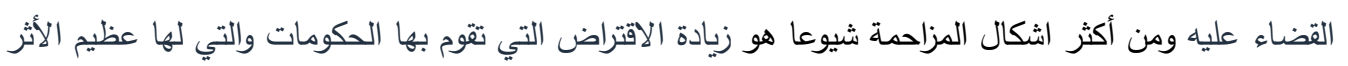

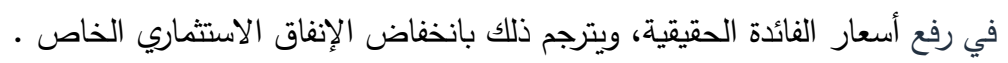


(Shetta \& Kamaly,2014,251-279) (Hauner,2009,171-183) banking Hypothesis) على تقويض جزء من كفاءة وإمكانية الجهاز المصرفي على منح الائتمان إلى القطاع الخاص الذي يعد

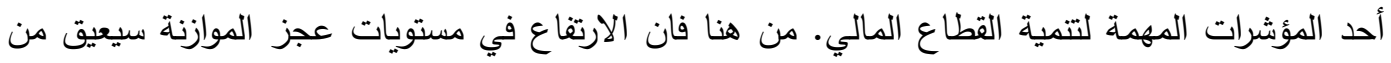

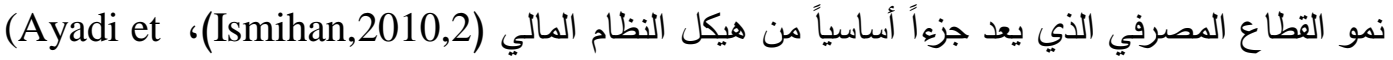
(Janda \& (İlgün,2016,284) ‘(Andrés et al.,2016,1)، al.,2013,4)

.Kravtsov,2017,10),

في مقابل ذلك يشير Kutivadze (2011), Kumhof \& Tanner (2005) إلى أن الأدوات الحكومية من سندات واذونات خزانة والتي يطلق عليها عادة بالموجودات الآمنة (Safe Asset) ونتيجة لتمتع أسواقها بالاستقرار النسبي وقدرتها على تسهيل عمل الوساطة المالية يمكن أن تسهم وبفاعلية في تطوير وتتمية الاسواق المالية وبالتالي القطاع المالي.

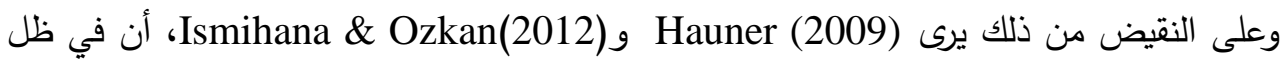
هيمنة الحكومة كمقترض رئيس في سوق الأوراق المالية المحلية، واستمرارية عجز الموازنة العامة وارتفاع مستوياته، يبدأ التأثير السلبي لارتفاع الدين العام والذي يمثل مقياساً رئيساً للتنمية المالية نتيجة لما يولدها

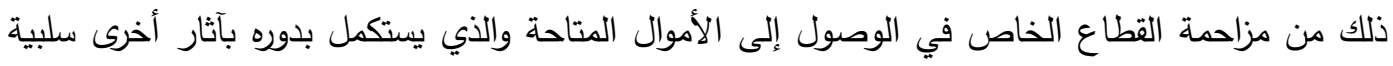
الأثر على النشاط الاقتصادي.

وفي اتجاه آخر لتحليل آلية التأثير غير المباشر لعجز الموازنة العامة على تطور القطاع المالي

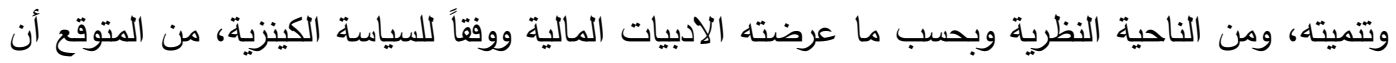
المستويات المرتفعة من الانفاق الحكومي، وعبر التغيرات الجوهرية في أسعار الفائدة وأسعار السلع النهائية

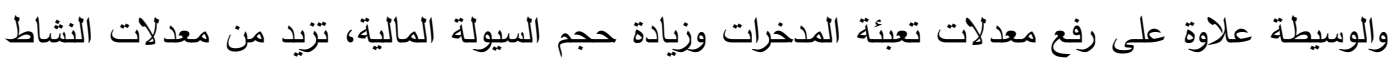

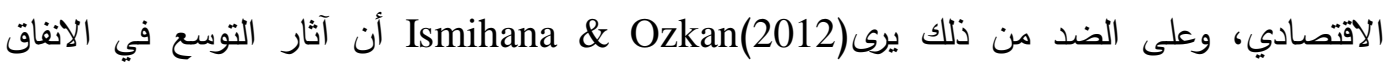
الحكومي قد تكون انكماشية ، لأن الذي يحدد تلك الاثار هو مستوى تطور سوق الدين والجمهور وهيكل

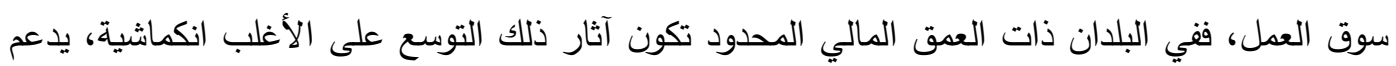
ذلك القول Christensen(2005 و Caballero and Krishnamurthy (2004) أن زيادة الانفاق الحكومي في مثل هكذا اقتصادات يزاحم استثمار القطاع الخاص عبر تبني سياسة مالية توسعية تؤدي إلى الى انخفاض قيمة وجودة الاوراق الحكومية بسبب انخفاض السيولة لدى الدولة.

r. أنموذج البنك الكسول هو إمكانية خلق خطر اخلاقي ناجم عن ارتفاع درجة الإقراض للحكومة بما يؤدي بالتالي

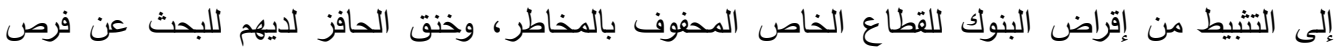
استثمارية مربحة جديدة في القطاع الخاص. ع. ـ يُعرَّف العمق المالي بأنه حجم الأموال المتاحة للتمويل الحكومي والقطاع الخاص 
في ضوء ما تقدم، ونظراً لما يمكن أن يحدثه عجز الموازنة العامة، بعدِه أحد متغيرات مصفوفة

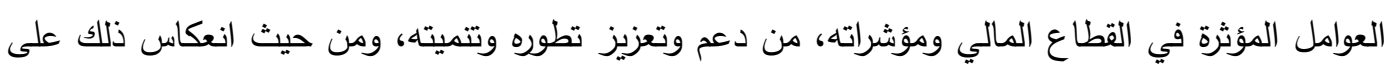

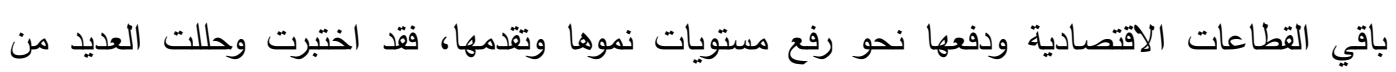

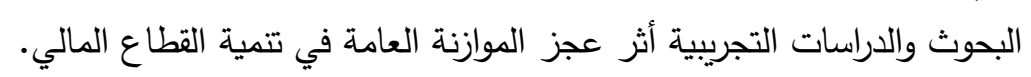
• مشكلة البحث

تخضع مستويات تطور القطاع المالي في بلدان العالم ولا سيما النامية منها لتأثير عجز الموازنة

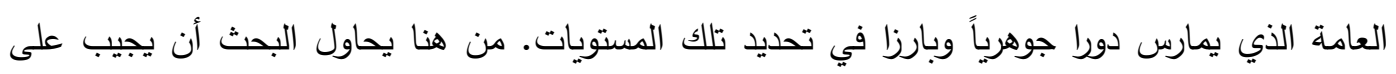

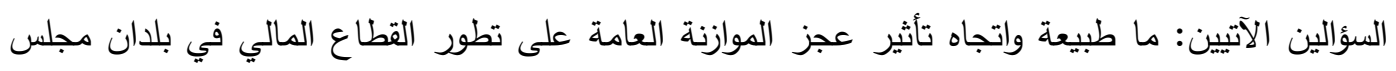

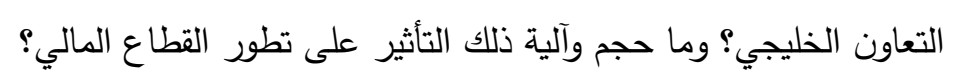
• مان البحث

لقد سعت الدراسة إلى تقديم صورة واضحة وشاملة عن الأطر النظرية والدراسات التجرببية التي

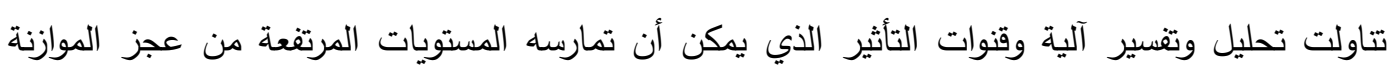

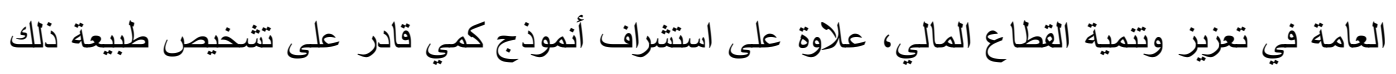
التأثير وقيمته واتجاهه لدول مجلس التعاون الخليجي وللمدة (2005-2017).

$$
\text { • - مرضية البحث }
$$

انطلق البحث من فرضية أساسية مفادها خضوع التطور المالي لتأثير عجز الموازنة العامة في مستوياته، ويتباين في طبيعة وحجم واتجاه ذلك التأثير .

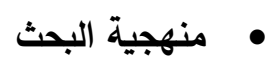

بغية بلوغ أهداف البحث واختبار فرضياته تم اعتماد المنهجية المستتدة إلى الربط بين اتجاهين

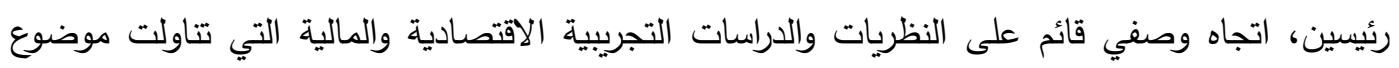
البحث بهدف تحديد ورصد وتثخيص أثر عجز الموازنة العامة في تعزيز وتتمية القطاع المالي، واتجاه ثاني يتبنى الاستعانة بأساليب الاقتصاد القياسي الحديثة المتمثلة بمنهجية الانحدار التجميعي (PRM)

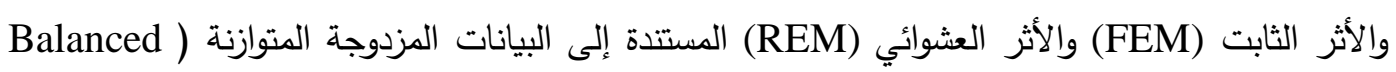
(Panel Data مجلس التعاون الخليجي. 


$$
\text { • حدود البحث }
$$

لقد اتخذ البحث، وللابتعاد عما طرحته الدراسات التجريبية السابقة، من دول مجلس التعاون الخليجي عينة لتطبيقاته، باعتبارها مكوناً اقتصادياً متقارباً ولحد بعيد من حيث لانيات خصائصه الاقتصادية والسياسية والاجتماعية r. (الحدود الزمانية

غطت فترة زمنية شهدت العديد من التغيرات السياسية والاقتصادية ورافقت الازمة المالية العالمية A . . r وما تبعها من تباطؤ نمو الاقتصاد العالمي إلى الانهيار الحاد في أسعار النفط الداعم لعجز الموازنة

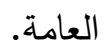

\section{1. مراجعة الأدبيات ذات العلاقة}

لم يكن تثخيص أثر عجز الموازنة العامة في تتمية وتطور القطاع المالي والوقوف على طبيعة

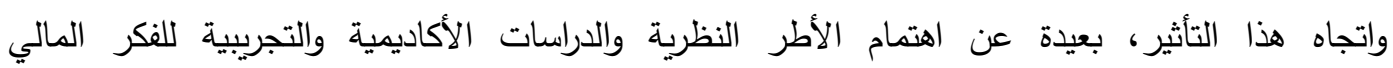

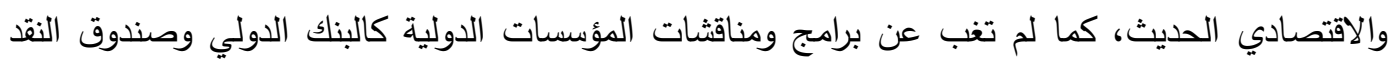
الدولي. فقد أجريت العديد من البحوث الدراسات التي تتاولت ذلك الأثر ولعينات ودول مختلفة، يمكن استعراض أهمها وعلى النحو الآتي: تناقش دراسة Kumhof and Tanner (2005 سلوك البنوك المحلية في اختيارها الإفراط في الاقراض الحكومي، والمنطلق من كونها تعتبر أن الإقراض الخاص هو البديل الأكثر خطورة في ظل

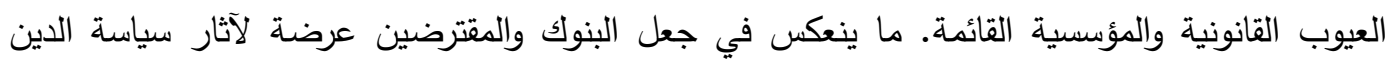
الحكومية. باستخدام منهجية VAR للتكامل المشترك في باكستان للفترة (2000-1964)، وأظهرت النتائج الأثر الكبير للاستثمار الحكومي على الاستثمار الخاص، ومن ثم تؤدي هذه الاستثمارات دورًا حيويًا في تعزيز النمو الاقتصادي. أجرت دراسة (2007) . Ardagna et al مسحاً لستة عشر دولة من دول منظمة التعاون الاقتصادي والتتمية OECD وباستخدام (VAR) أظهرت النتائج أن ازدياد الدين الحكومي من البنوك قد أدى إلى رفع سعر الفائدة في بلدان منظمة التعاون الاقتصادي والتتمية، أما في المدى الطويل فهذا الارتفاع في سعر الفائدة يؤدي إلى انخفاض النفي الاستثمار الخاص الذي يعد بمثابة جوهر النمو الاقتصادي لتلك الدول. وذلك بسبب لجوء الحكومة عندما تواجه عجزًا في الايرادات إلى الاقتراض، مما يخلق مشكلة مزاحمة المستثمرين من القطاع الخاص الذين الذين يرغبون في الاقتراض من القطاع المصرفي. وهذا ما يحدث عادة في البلدان النامية. في دراسة (2009) مول محددات الاستثمار الخاص في غانا، وباستخدام منهجية الانحدار الذاتي للفجوات الزمنية الموزعة (ARDL) للمدة (2005-1972)، أظهرت النتائج وجود 
علاقة طويلة الاجل، فضلا عن إلى العلاقة قصيرة الاجل بين الاستثمار الخاص والانفاق الحكومي، كما

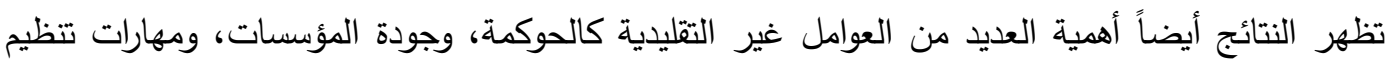
المشاريع في رفع حجم الاستثمار الخاص. مقابل هامشية وانحسار تأثير العوامل التقليدية الذي قد ودئ يصل

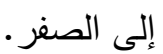

اهتمت دراسة Nejad (2010) بمحدات التتمية المالية وتقييم آثار السياسات المالية القمعية على

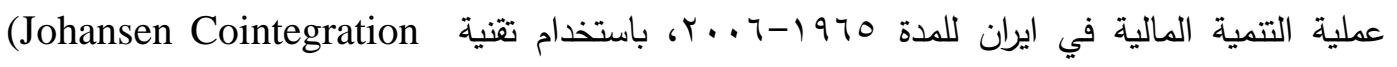
لبيانات السلسلة الزمنية. وأكدت نتائج العمل التجريبي على أن كلاً من الانفتاح التجاري

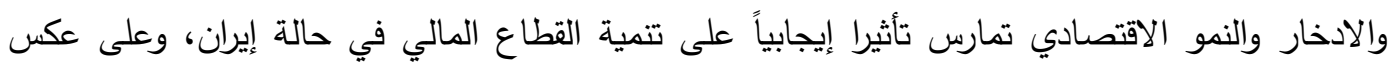

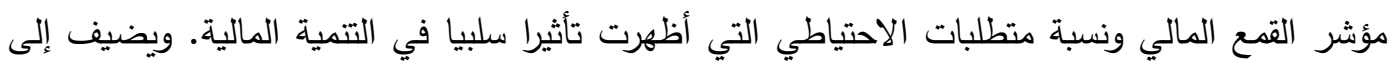
ذلك بالقول إن النتيجة المثيرة للاهتمام هي الأثر الايجابي للتضخم على التتمية المالية في حالة إيران، وهو

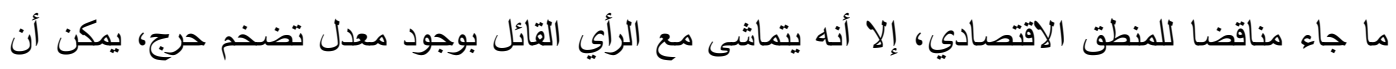
يشجع النشاط الحقيقي ويعزز التمية المالية بدلاً من عرقلتها. في دراسة (2013) Takyi \& Obeng حول محددات التتمية المالية في غانا وباستخدام منهجية الانحدار الذاتي للفجوات الزمنية الموزعة (ARDL) وباعتماد البيانات الفصلية للمدة 1988-2010،

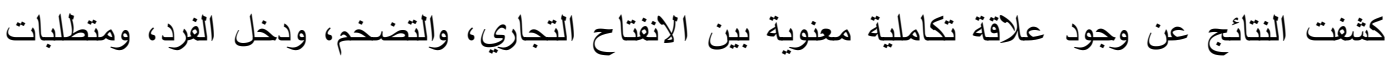
الاحتياطي والاقتراض الحكومي، علاوة على الدور المهم الذي يمكن أن يؤديه الانفتاح التجاري ودخل ولنه الفرد في تحديد مستوى التنمية المالية في غانا. فبينما كان للتضخم ومعدل الفائدة ومتطلبات الاحتياطي آثار سلبية وذات دلالة إحصائية على التتمية المالية في المدى القصير والطويل، فثل الإقراض الحكومي في في فئي

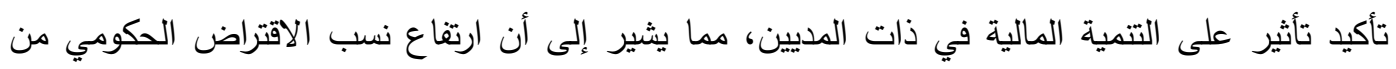

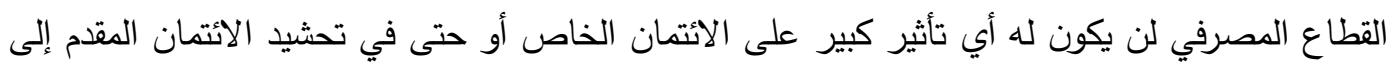
القطاع الخاص.

تناولت دراسة (2016) Ali, et al. تأثير الاقتراض الحكومي من البنك المركزي والبنوك التجارية على التتمية المالية. باستخدام الاقتراض الحكومي كديون محلية عامة في حين استخدم الايتمان المقدم إلى

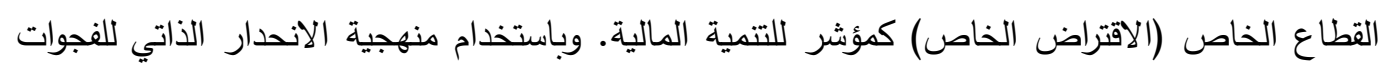

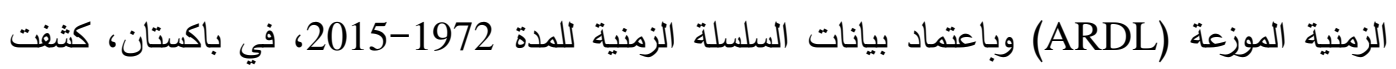

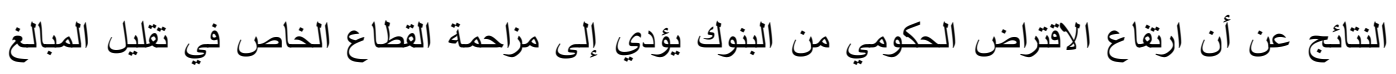
المتاحة للاقتراض، ما ينعكس في انخفاض حجم استثمار القطاع الخاص، علاوة على وجود بعض العضى العوامل الأخرى المؤثرة أيضا على حجم اقتراض القطاع الخاص مثل الضرائب والمدخرات والتضخم. حللت دراسة (2009) بussain et al بالعلاقة طويلة الأجل بين استثمار القطاع الخاص

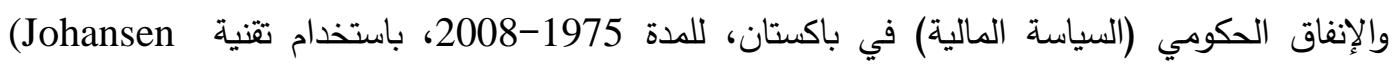


لبيانات السلسلة الزمنية. وأكدت نتائج العمل التجريبي على أن هناك

$$
\text { ارتباطًا طويل الأجل بين متغيرات الدراسة. }
$$

تتاولت دراسة Raymond Alenoghena (2018) آثار تمويل العجز المالي من المصادر المحلية على تتمية الأسواق المالية في نيجيريا، وتحديد ماهية وأهمية العلاقة بين خيارات تمويل العجز المالي وتطوير الأسواق المالية للبلاد على المديين القصير والطويل. بالإضافة إلى تثخيص أثر الدين الدين

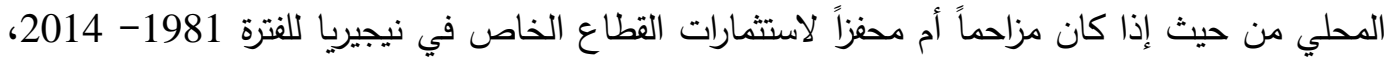

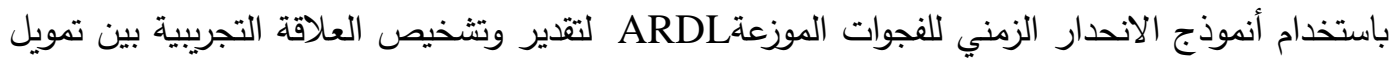
العجز المالي وتتمية الأسواق المالية.. وأظهرت النتائج وجود ارتباط طويل الأمد يتجه من العجز المالي إلى الى العي تطوير الأسواق المالية. وبشكل أكثر تحديدًا، يؤثر عجز الموازنة والديون المحلية والإنفاق الحكومي بشكل كبير وسلبي على تطوير الأسواق المالية النيجيرية. علاوة على الأثر السلبي لعجز الموازنة العامة للدولة على تتمية القطاع المالي على المدى الطويل. ويضيف إلى ذلك بالقول إن النتيجة المثيرة للاهتمام هي التي الأثر الكبير والسلبي للدين المحلي على اعتماد إقراض استثمارات القطاع الخاص في نيجيريا، وهو ما جاء مواقفاً للمنطق الاقتصادي القائل بأن الدين العام المحلي يزاحم استثمارات القطاع الخاص. وتثير نتائج الدراسة إلى أن السياسة المالية في نيجيريا لها دور قوي في استقرار الاقتصاد الكلي. ويُنصح الحكومة لعادئ بتقليص العجز الهائل والمستمر في الميزانية. إذ لابد من أن تؤدي السياسات المالية دوراً أكثر حيادية في تحفيز الاستثمار وريادة الأعمال في القطاع الخاص في الاقتصاد النيجيري. ويحاول الجدول (1) استعراض بعض نتائج الدراسات التي اهتمت بأثر عجز الموازنة العامة على العى تطور وتتمية القطاع المالي.

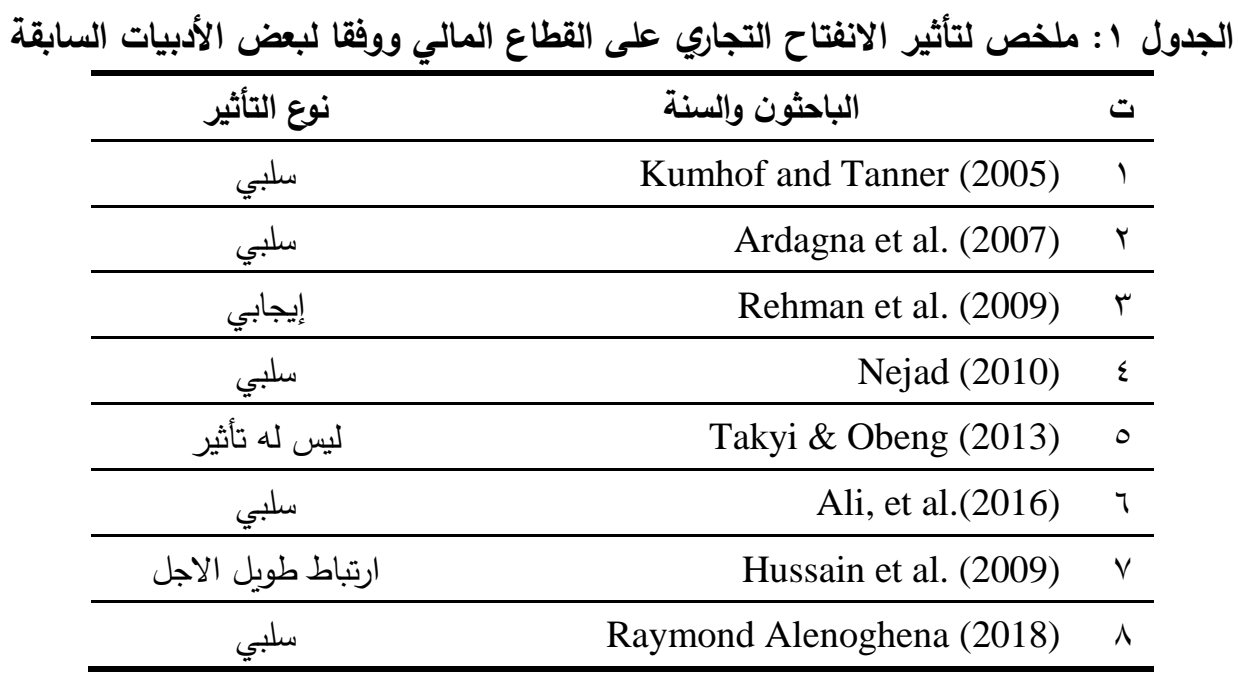

الجدول من عمل الباحثة بالاعتماد على الدراسات السابقة 


\section{r. المنهجية التجريبية (Empirical Methodology)}

في ضوء ما تقدم من دراسات سابقة للباحثين الاقتصاديين والماليين، واستتادا إلى الأطر النظرية

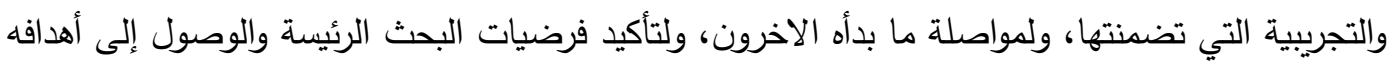
الأساسية، وبهدف تصميم أنموذج كمي لأثر واتجاه علاقة مصفوفة متغيرات الدراسة (المتغيرات التوضيحية المتمثلة: بعجز الموازنة العامة، والنمو الاقتصادي، والتضخم) في تتمية القطاع المالي ومؤشراته (المتغير

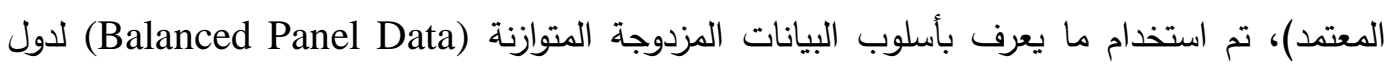
مجلس التعاون الخليجي وللفترة (2005-2017) وبواقع عدد مشاهدات السلسلة الزمنية (†^) مشاهدة 1 ا مصادر البيانات

بهدف توحيد مصادر البيانات وتجنب تباينها واختلافها، اشتملت مصادر البيانات لدول مجلس

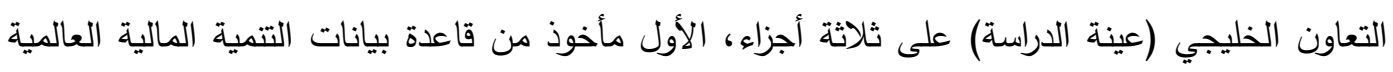
(World Bank) الصادرة عن البنك الدولي من (Global Financial Development Database) والخاص ببيانات مؤشرات التتمية المالية. في حين يستند الثاني والثالث على التوالي إلى قاعدة بيانات البنك الدولي والمعنية بمؤشرات التتمية العالمية (World Development Indicators)، وقاعدة بيانات صندوق النقد الدولي الخاصة بالإحصاءات المالية الدولية (International Financial Statistics) للوصول إلى بيانات المتغيرات التفسيرية (عجز الموازنة العامة، والنمو الاقتصادي، والتضخم)

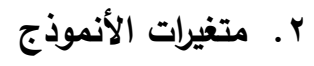
أ. أم المتغيرات المعتمدة الائمودة

انطلاقاً من البحوث والدراسات التجريبية مثل (2019) Svirydzenka Gupta \& Mahakud (2016)، (2012) . Čihák et al والمستندة لآراء خبراء البنك الدولي وصندوق النقد الدولي في تمييز مؤشرات تتمية القطاع المالي، تم اعتماد مؤشرين للتعبير عن تتمية الأسواق المالية، ومؤشرين للتعبير عن تتمية القطاع المصرفي، والتي عدت أكثرها شيوعًا واستخدامًا من قبل تلك الدراسات وعلى التمات النحو الآتي:

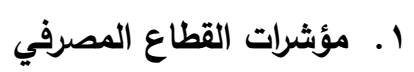

الائتمان المصرفي الممنوح للقطاع الخاص بوصفه نسبة من الناتج المحلي الإجمالي (BC):

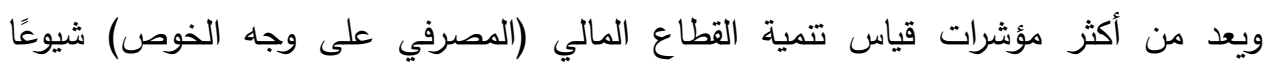
واستخدامًا في الدراسات الاقتصادية والمالية، نظرا لقدرته على التعبير عن حجم الوساطة المالية

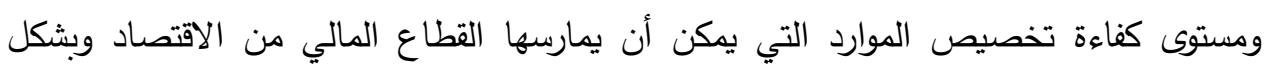

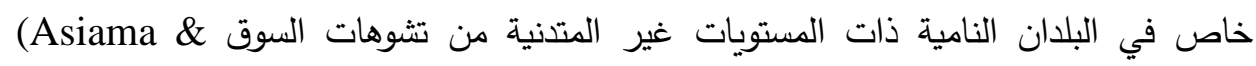

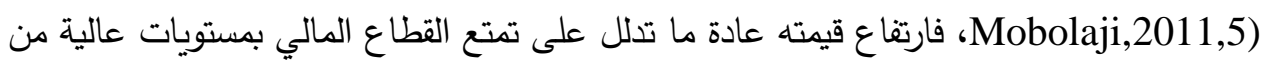
الفاعلية والتطور (Asiama \& Mobolaji,2011,5). (Ross et al., 2000,31-77) . 
إجمالي ودائع القطاع المالي بوصفه نسبة من الناتج المحلي الإجمالي (TD): وتثكل الودائع

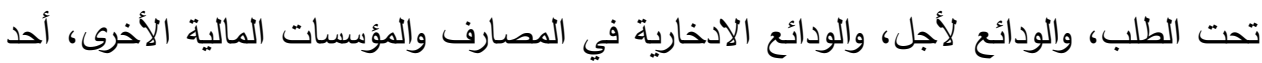
أكثر المؤشرات المالية واقعية ودقة لقياس مدى تطور القطاع المالي بعد تتسيبه إلى الناتج المحلي الإجمالي وخاصة في البلدان النامية التي تتميز بارتفاع مستويات العملة المتداولة إلى إجمالي

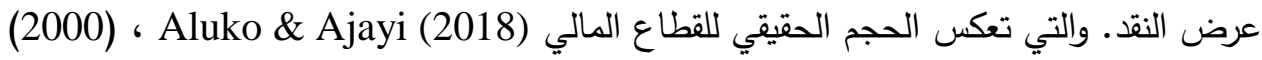
Ghali (1999), Ardicad \& Damar Demetriades \& Hussein (1996) ، Xu (2006)، بعدها تعبيرا عن ميل الوحدات الاقتصادية باتجاه التعامل مع الودائع المصرفية من هن جانب، ورغبة وقدرة المؤسسات المالية في تعبئة الموارد المالية وإعادة تخصصيها بين الوحدات الاقتصادية من جانب آخر، فارتفاع نسبتها إلى الناتج المحلي الإجمالي، إنما يعكس مستوى

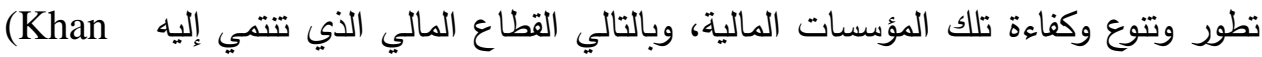
.(Gregorio \& Guidotti,1995,433-448) ، \& Senhadji,2003,89-110)

r ب ـ مؤشرات الأسواق المالية

نسبة القيمة السوقية إلى الناتج المحلي الإجمالي (SMC): والذي يعبر عن مستوى العمق المالي الذي تتمتع به أسواق الأوراق المالية (Levine \& Zervos ,1998, 537-558) ويشير بصورة الإئه خاصة إلى حجم السيولة التي تمتلكها تلك الأسواق-Nazir,Nawaz \& Gilani,2010,3473) (3479. فارتفاع قيمة المؤشر وما يعكسه من ارتفاع سيولة أسواق الأوراق المالية عادة ما يفسر

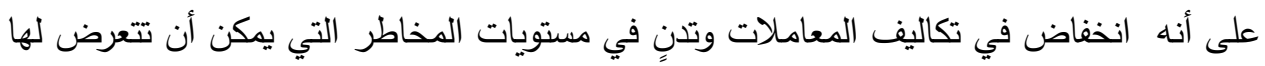
الاستثمارات المتتوعة وجاذبية اكبر لوحدات الفائض المالي لاستبدال مدخراتهم النقدية إلى فئي موجودات مالية قادرة على توليد عوائد مستقبلية، طالما ليس هناك ما يمنع من استعادة تلك لكاتك

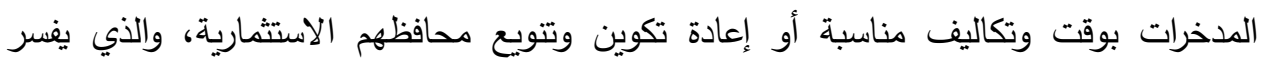

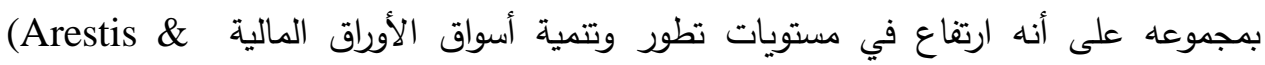
Ognaligui ،(Demirguc-Kunt \& Levine,1996,229) Demetriades ,1997,785)

.(Ake \& ,2017,83) معدل دوران سوق الأسهح (الأسهم المتداولة إلى الرسملة) (SMT): يعبر هذا المؤشر عادة عن

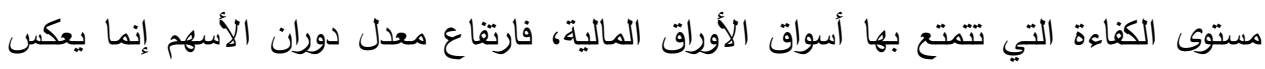

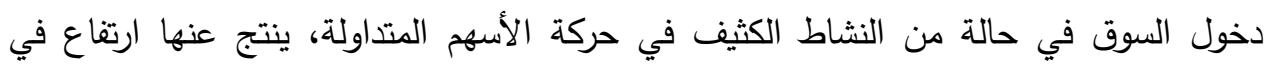

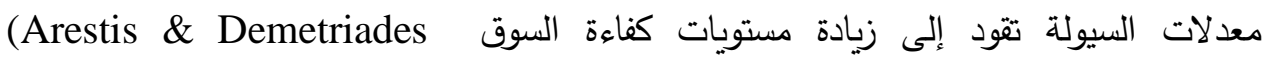
.(Balogu, Asso \& Hassan,2016,40) ،, 1997,785) 
ب. المتغيرات التوضيحية (المستقلة)؛ لتوضيح أثر عجز الموازنة العامة في تتمية القطاع المالي

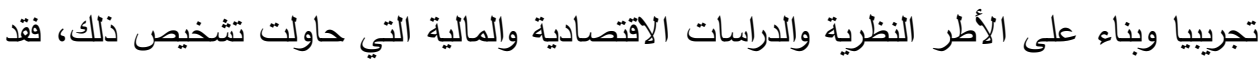

$$
\text { اعتمدت متغير : (2) }
$$

عجز الموازنة العامة (BUD): تم التعبير عن عجز الموازنة العامة بنسبة العجز في الموازنة

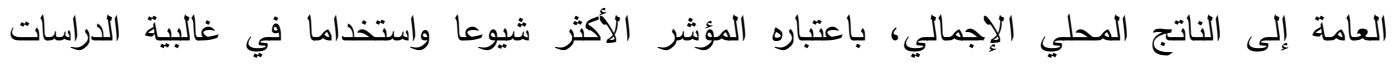

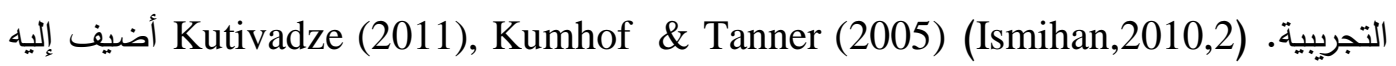
متغيران اقتصاديان آخران ضابطان (Control Variables) وبحسب الدراسات الاقتصادية والمالية يعدان

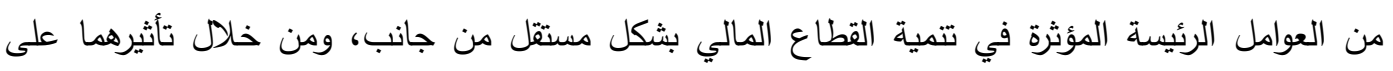

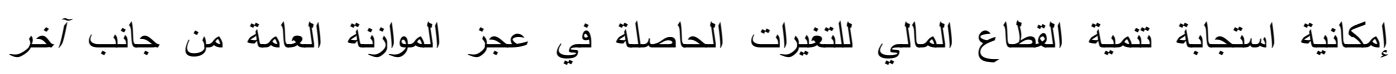
: (Ho \& Bernard,2018,6) ، (Huang, 2010,165) أ. النمو الاقتصادي (GDP): ويعبر عنه بمعدل التغير في حصة الفرد من الناتج المحلي الإجمالي

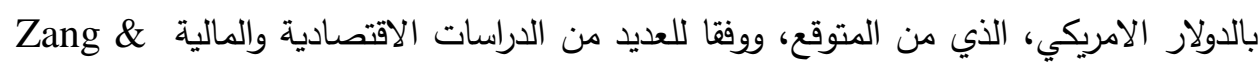
Kim (2007) Arestis \& Demetriades (1996), Baltagi et al. (2009), Law \& Habibullah (2009), Odhiambo (2012),Gozgor (2015),Peia \& Roszbach (2015), ب. التضخم (INF): تم التعبير عنه بالتغير السنوي في الرقم القياسي لأسعار المستهلك، والذي لتئي

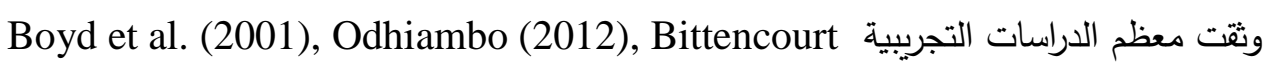
(2014), Ben \& Kandil (2008)، الآثار السلبية لارتفاع معدلاته على تتمية القطاع المالي وتطوره.

تأسيساً على ما تقدم يمكن صياغة نماذج التقدير على شكل أربعة دوال رياضية يُستتد عليها في

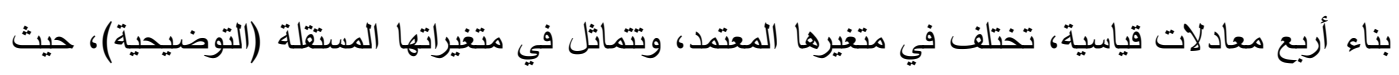
تعكس المعادلة الأولى أثر عجز الموازنة العامة والنمو الاقتصادي والتضخم في مؤشر تتمية القطاع المالي

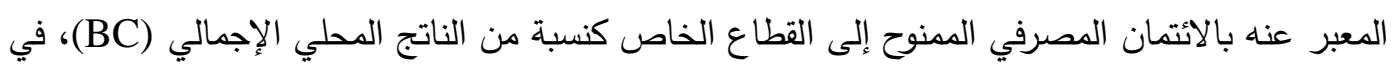
حين تبنى المعادلة الثانية لتبين أثر تلك المتغيرات التوضيحية في مؤشر تتمية القطاع المالي المتمثل

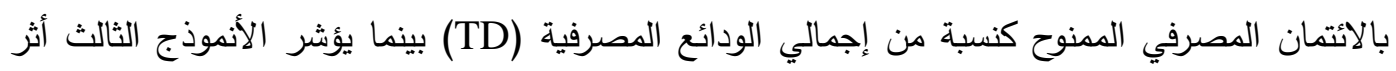

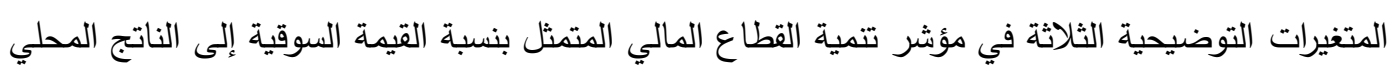

ع. حول مؤشرات المتغيرات التفسيرية المتمدة، والدراسات التجريبية التي اعتمدتها. راجع الملحق ( () ه. تسهم المتغيرات الضابطة (Control Variables) الداخلة في الأنموذج، وعبر تقليلها لنسب الأخطاء العشوائية

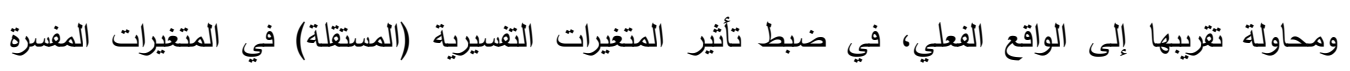
(التابعة)، ورفع مستوى دقة ووضوح الأنموذج. 
الإجمالي (SMC) وأخيراً توضح المعادلة الرابعة أثر المتغيرات التوضيحية المعتمدة في النماذج السابقة في مؤشر تتمية القطاع المالي المعبر عنه بمعدل دوران سوق الأسهح (SMT) (SMT)، وكما يأتي:

(1) $B C=F\{B U D, G R O, I N F\}$

(2)TD $=F\{B U D, G R O, I N F\}$

(3) $S M C=F\{B U D, G R O, I N F\}$

(4) $S M T=F\{B U D, G R O, I N F\}$

(1) $B C=\beta_{0}+\beta_{1} B U D+\beta_{2} G R O+\beta_{3} I N F+\varepsilon_{i}$

(2)TD $=\beta_{0}+\beta_{1} B U D+\beta_{2} G R O+\beta_{3} I N F+\varepsilon_{i}$

(3) $S M C=\beta_{0}+\beta_{1} B U D+\beta_{2} G R O+\beta_{3} I N F+\varepsilon_{i}$

(4) $S M T=\beta_{0}+\beta_{1} B U D+\beta_{2} G R O+\beta_{3} I N F+\varepsilon_{i}$

r. منهجية تقدير الأنموذج.

تكتسب البيانات المزدوجة (Panel Data) اهتماماً بالغاً في الآونة الاخيرة وبشكل خاص في الاصدي

الدراسات الاقتصادية والمالية، لما تتسم به من قدرة على الدمج بين خصائص كل من البيانات المقطعية

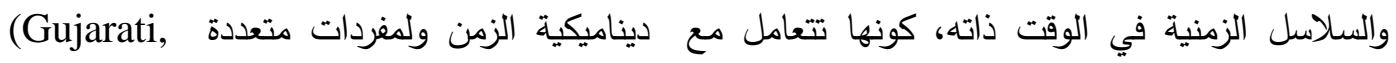
(2004,636 علاوة على معالجتها لمشكلة عدم التجانس أو التباين غير الواضح لمفردات العينة المقطعية، ومن ثم الابتعاد عن الوقوع في تقديرات متحيزة وغير متسقة (Reyna, 2007,2). ويتم التعامل مع (20) البيانات المزدوجة ووفقا لـ Green (2002) بثلاثة مناهج انحدار أساسية هي:

أ. أنموذج الانحدار التجميعي (Pooled Regression Model) (PRM) يعد أنموذج (PRM) من أبسط نماذج تحليل البيانات المزدوجة (panel Data) ، حيث يستتد

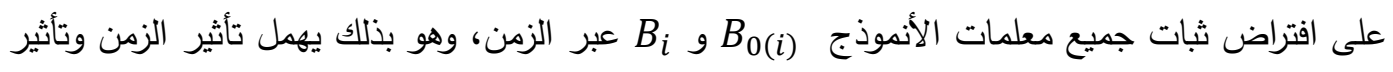

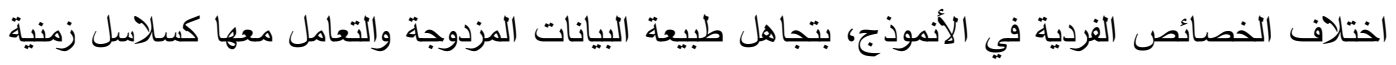
(Time Series) من أول مجموعة بيانات مقطعية وهكذا، فتتكون سلسلة زمنية بحجم مشاهدات مقداره (N * T) حيث

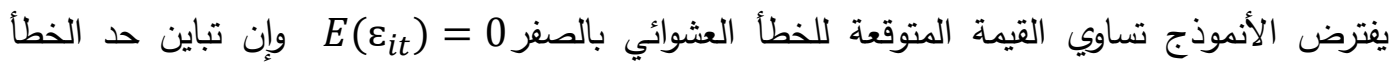
العشوائي ثابت الحصول على تقديرات تتصف بالاتساق (Consistent) والكفاءة (Efficient) شرط ثبات الأثر الفردي

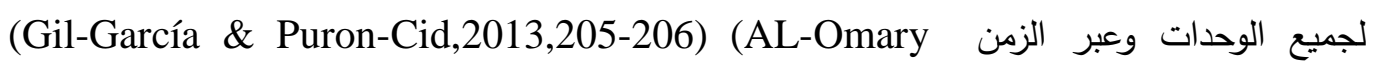
\&Hyt, 2016, 883)

$$
Y_{i t}=\alpha_{i}+\sum_{i=1}^{k} B_{j} X_{j(i t)}+\varepsilon_{i t} \quad i=1,2, \ldots \ldots ., N \quad t=1,2, \ldots \ldots \ldots, T
$$

TANMIYAT AL-RAFIDAIN (P-ISSN: 1609-591X; E-ISSN: 2664-276X) تنمية الرافدين 
ب. بموذج الأثر الثابت (Fixed Effect Model) (FEM) يستتد أنموذج الأثر الثابت (FEM) على افتراض وجود اختلافات غير مشاهدة وغير متجانسة

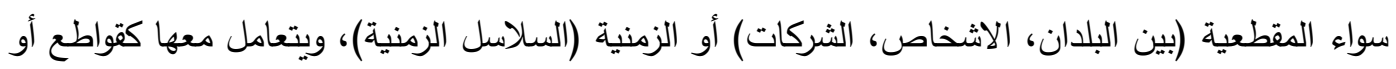
ثوابت (Constant)، أي يتقبل الأنموذج وجود ثوابت تتفاوت بحسب كل بلد (Cross Sectional) أو الو بحسب كل فترة زمنية (Time series) بهدف استكثاف الاثار غير المشاهدة والمؤثرة في المتغير المعتمد، ولتقدير هذه الثوابت تستخدم طريقة المربعات الصغرى للمتغيرات الوهمية (Least-Squares (LSDV) (n-1) لتمثيل الدول والتي تحتوي على متغيرات وهمية بعدد Dummy Variable Model) لتمثيل السنوات، وفي ضوء ما سبق فإن هذا الأنموذج يعكس الاختلافات والفروق بين الوحدات المقطعية

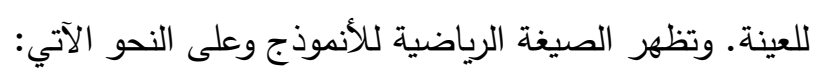
$Y_{i t}=\alpha_{i}+\sum_{i=1}^{k} B_{j} X_{j(i t)}+\varepsilon_{i t} \quad i=1,2, \ldots ., N \quad t=1,2, \ldots \ldots, T$

$$
\alpha_{i}=\alpha_{0}+u_{i}
$$

حيث تتوزع الاخطاء العشوائية طبيعيا حول الوسط بمتوسط صفر ، وتباين ثابت .(AL-Iraqi, 2018, 84-85) (Clark et al.,2010,7-8)

ت. أنموذج الأثر العشوائي(Random Effect Model) (REF) على خلاف أنموذج الأثر الثابت (FEM)، يتعامل أنموذج الأثر العشوائي (REF) معائ (RE) مع الاثار

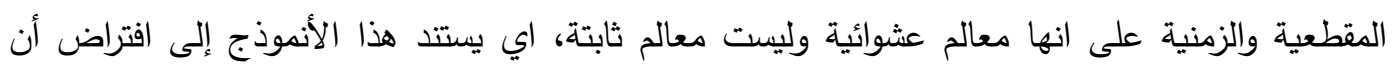

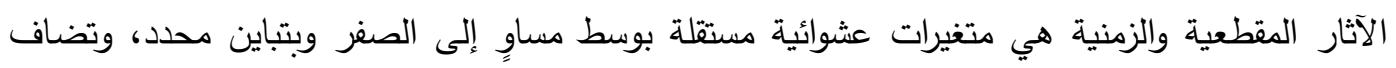

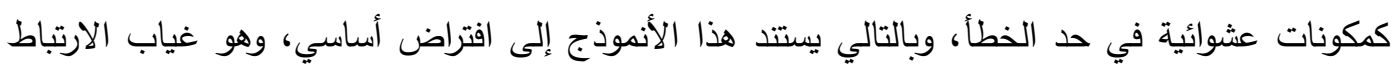

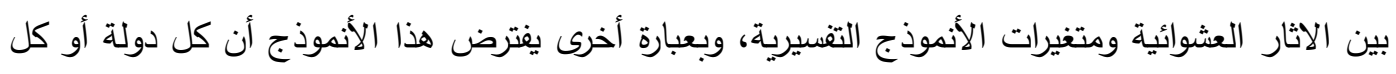

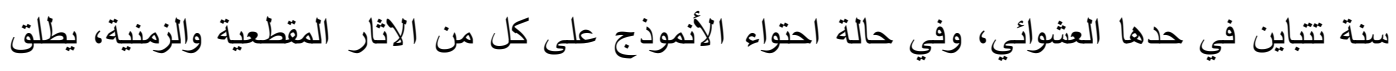

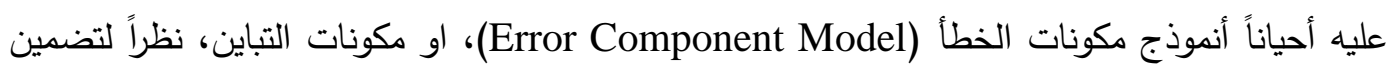

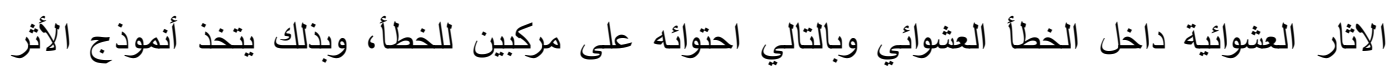
$Y_{i t}=\alpha_{i}+\sum_{i=1}^{k} B_{j} X_{j(i t)}+\epsilon_{i t} \quad i=1,2, \ldots ., N \quad t=1,2, \ldots, T$ العشوائي (REM) الصيغة الآتية: الصئية $\epsilon_{i t}=u_{i}+\varepsilon_{i t}$ بافتراض أن حد الخطأ المركب $\epsilon_{i t}$ يمتلك خصائص الأخطاء العشوائية الأخرى نفسها من حيث أن قيمته المتوقعة مساوية للصفر ( 
وفي ضوء ما سبق، من تغير الخطأ العشوائي إلى خطا مركب في عملية تقدير معلمات الأنموذج،

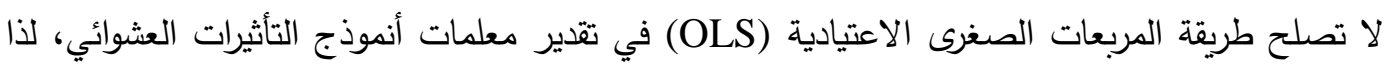

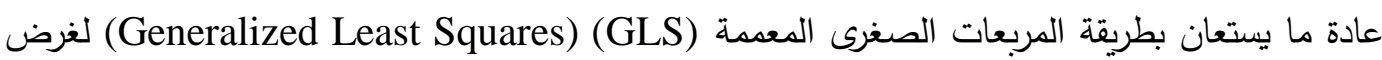

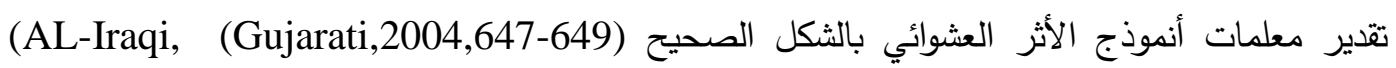
2018, 85)

ولاختيار الأنموذج الأكثر ملاعمة من ضمن نماذج البيانات المزدوجة (Panel Data) الثلاثة

$$
\text { السابقة، يتم إجراء اختبارين احصائيين تشخيصيين يتمان عبر مرحلتين: }
$$

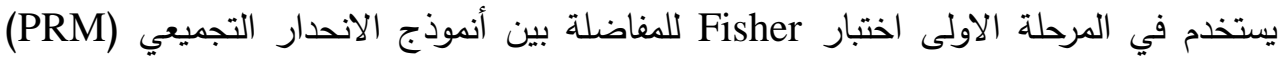
وأنموذج الأثر الثابت (FEM) في التقدير . فاذا اشارات النتائج إلى أفضلية وملاءمة أنموذج الانحدار لإندار

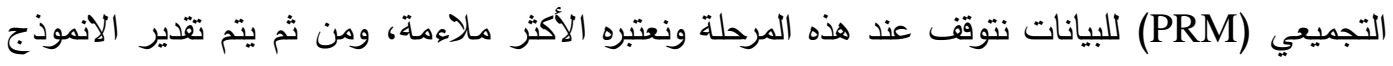

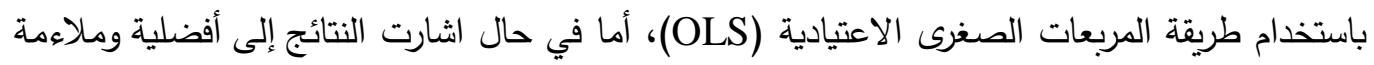

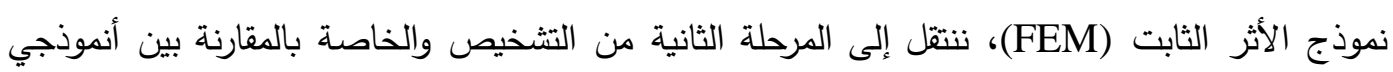

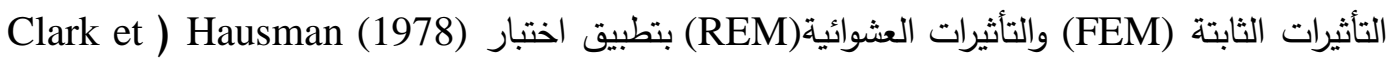

$$
\text { .(al.,2010,25-27 }
$$

$$
\text { ؛. تقدير النماذج }
$$

للوصول إلى نتائج وتقديرات لأثر عجز الموازنة العامة على تتمية القطاع المالي في بلدان مجلس

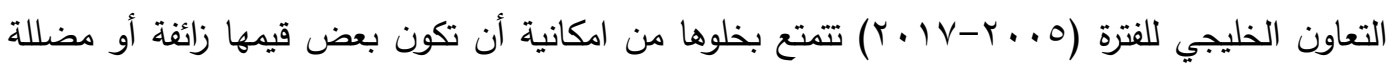

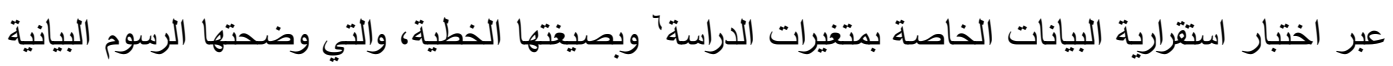

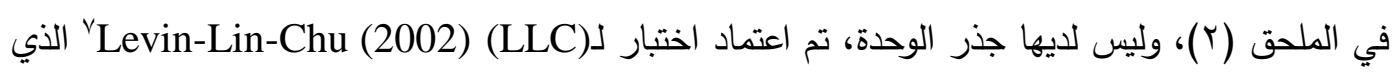
تم ادراج نتائجه في الملحق (r)، والتي أكدت على استقرارية متغيرات الدراسة في المستوى، باستثناء متغيري الائتمان المصرفي الممنوح للقطاع الخاص إلى الناتج المحلي الإجمالي (BC)، وإجمالي ودائع

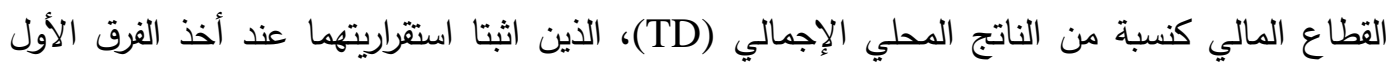

بناءً على ما تقدم من معطيات، وباعتماد نتائج اختبار (1960) Chow-Test التي عرضت في

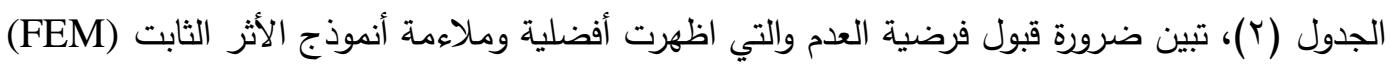
مقارنة بأنموذج الانحدار التجميعي (PRM) في تحليل البيانات المزدوجة (Panel Data) للنماذج الاربعة.

T. للاطلاع على الوصف الإحصائي لمتغيرات الدراسة من حيث الوسط الحسابي، والوسيط، الانحراف المعياري،

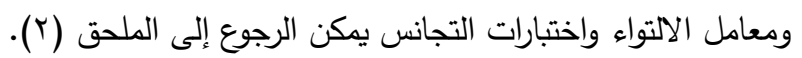

7 . Levin, A., Lin, C.F., Chu, C. (2002) .Unit root tests in panel data: Asymptotic and Finite Sample Properties. Journal of Econometrics, 108:1-24. 
الجدول r : اختبار Chow-Test للنماذج الاربعة

\section{Redundant Fixed Effects Tests \\ Equation: Untitled \\ Test cross-section fixed effects}

\begin{tabular}{|c|c|c|c|}
\hline \multicolumn{4}{|c|}{ للأنموذج الاول BC } \\
\hline Effects Test & Statistic & d.f. & Prob. \\
\hline Cross-section F & 12.445768 & $(5,70)$ & 0.0000 \\
\hline Cross-section Chi-square & 49.611029 & 5 & 0.0000 \\
\hline \multicolumn{4}{|c|}{ للأنموذج الثاني TDow-Test TD } \\
\hline Effects Test & Statistic & d.f. & Prob. \\
\hline Cross-section F & 32.420919 & $(5,70)$ & 0.0000 \\
\hline Cross-section Chi-square & 93.498043 & 5 & 0.0000 \\
\hline \multicolumn{4}{|c|}{ للأنموذج الثالث IS IS-Test|ختبار IChow-T } \\
\hline Effects Test & Statistic & d.f. & Prob. \\
\hline Cross-section F & 12.912926 & $(5,71)$ & 0.0000 \\
\hline Cross-section Chi-square & 50.447952 & 5 & 0.0000 \\
\hline \multicolumn{4}{|c|}{ SPV للأنموذج الزابع Chow-Test|ختبار SPV } \\
\hline Effects Test & Statistic & d.f. & Prob. \\
\hline Cross-section F & 24.302382 & $(5,71)$ & 0.0000 \\
\hline Cross-section Chi-square & 77.803296 & 5 & 0.0000 \\
\hline
\end{tabular}

الجدول من إعداد الباحثين بالاعتماد على مخرجات برنامج Eviews10

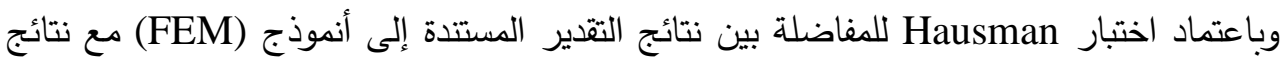

أنموذج (REM) للنماذج الاربعة، والمثبتة نتائجه في الجدول (3)، تبين قبول الفرضية البديلة وما تفترضه

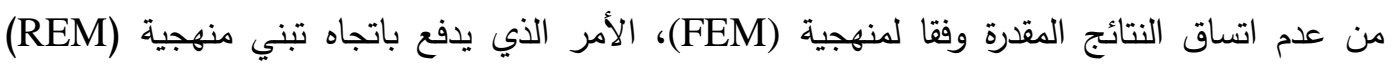
وللنماذج الاربعة.

\section{الجدول ץ: اختبار Hausman للنماذج الاربعة}

\begin{tabular}{|c|c|c|c|}
\hline \multicolumn{4}{|c|}{$\begin{array}{l}\text { Correlated Random Effects - Hausman Test } \\
\text { Equation: Untitled } \\
\text { Test cross-section random effects }\end{array}$} \\
\hline \multicolumn{4}{|c|}{ للأنموذج الاولHausman|ختبار } \\
\hline Test Summary & Summary Chi-Sq. Statisti & Chi-Sq. d.f & Prob. \\
\hline $\begin{array}{l}\text { Cross-section } \\
\text { random }\end{array}$ & 0.307521 & 2 & 0.8575 \\
\hline \multicolumn{4}{|c|}{ للأنموذج الثاني Hausman اختبار } \\
\hline Test Summary & Summary Chi-Sq. Statisti & Chi-Sq. d.f & Prob. \\
\hline $\begin{array}{l}\text { Cross-section } \\
\text { random }\end{array}$ & 0.714982 & 2 & 0.6994 \\
\hline \multicolumn{4}{|c|}{ للأنموذج الثالث Hausman اختبار } \\
\hline
\end{tabular}

TANMIYAT AL-RAFIDAIN (P-ISSN: 1609-591X; E-ISSN: 2664-276X) تنمية الرافدين 


\begin{tabular}{|c|c|c|c|}
\hline \multicolumn{4}{|c|}{$\begin{array}{l}\text { Correlated Random Effects - Hausman Test } \\
\text { Equation: Untitled } \\
\text { Test cross-section random effects }\end{array}$} \\
\hline Test Summary & Summary Chi-Sq. Statisti & Chi-Sq. d.f & Prob. \\
\hline $\begin{array}{l}\text { Cross-section } \\
\text { random }\end{array}$ & 0.163519 & 1 & 0.6859 \\
\hline \multicolumn{4}{|c|}{ للأنموذج الرابع Hausman اختبار } \\
\hline Test Summary & Summary Chi-Sq. Statisti & Chi-Sq. d.f & Prob. \\
\hline $\begin{array}{l}\text { Cross-section } \\
\text { random }\end{array}$ & 0.694833 & 1 & 0.4045 \\
\hline
\end{tabular}

الجدول من إعداد الباحثين بالاعتماد على مخرجات برنامج Eviews10

بناء على ما تقدم من معطيات، وباعتماد منهجية الأثر العشوائي (REM) في تقدير أثر عجز الموازنة العامة (BUD) والمتغيرات الضابطة التضخم (INF) والنمو الاقتصادي (GDP) في مؤشرات التتمية المالية المعبر عنها في الأنموذج الأول بالائتمان المصرفي الممنوح للقطاع الخاص كنسبة من الناتج المحلي الإجمالي (BC)، وفي الأنموذج الثاني بالائتمان المصرفي المنوح كنسبة من إجمالي الودائع المصرفية (TD) وفي الأنموذج الثالث بنسبة القيمة السوقية إلى الناتج المحلي الإجمالي (SMC) وفي الإني

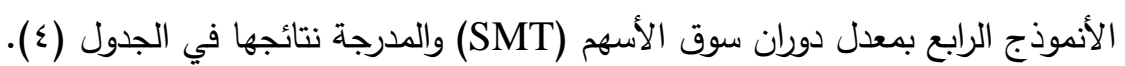

الجدول ؛ : أثر عجز الموازنة العامة والمتغيرات الضابطة في مؤثر التنمية المالية

Method: Panel EGLS (Cross-section random effects)

Sample: 20052017

Periods included: 13

Cross-sections included: 6

Total panel (balanced) observations: 78

Swamy and Arora estimator of component variances

$$
\text { الأنموذج الاول }
$$

أثر عجز الموازنة العامة والمتغيرات الضابطة في مؤشر التنمية المالية (BC)

\begin{tabular}{|c|c|c|c|c|}
\hline Variable & Coefficient & Std. Error & t-Statistic & Prob. \\
\hline BUD & -80.75528 & 13.17235 & -6.130666 & 0.0000 \\
\hline GRO & -1.233333 & 0.263095 & -4.687785 & 0.0000 \\
\hline $\mathrm{C}$ & 63.08515 & 5.125528 & 12.30803 & 0.0000 \\
\hline \multicolumn{5}{|c|}{ Weighted Statistics } \\
\hline R-squared & 0.514208 & \multicolumn{2}{|c|}{ F-statistic } & 39.69359 \\
\hline Adjusted R-squared & 0.501254 & \multicolumn{2}{|c|}{ Prob. (F-statistic) } & 0.000000 \\
\hline Durbin-Watson stat & 0.919551 & & & \\
\hline \multicolumn{5}{|c|}{ 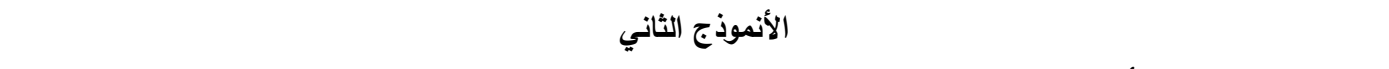 } \\
\hline \multicolumn{5}{|c|}{ أثر عجز الموازنة العامة وإلمتغيرات الضابطة في مؤشر التنمية المالية (TD) } \\
\hline Variable & Coefficient & Std. Error & t-Statistic & Prob. \\
\hline BUD & -72.26684 & 13.34117 & -5.416831 & 0.0000 \\
\hline GRO & -1.337881 & 0.266865 & -5.013324 & 0.0000 \\
\hline
\end{tabular}

TANMIYAT AL-RAFIDAIN (P-ISSN: 1609-591X; E-ISSN: 2664-276X) تنمية الرافدين 


\begin{tabular}{|c|c|c|c|c|}
\hline $\mathrm{C}$ & 59.90579 & 7.891725 & 7.590962 & 0.0000 \\
\hline \multicolumn{5}{|c|}{ Weighted Statistics } \\
\hline R-squared & 0.493570 & \multicolumn{2}{|c|}{ F-statistic } & 36.54781 \\
\hline Adjusted R-squared & 0.480066 & \multicolumn{2}{|c|}{ Prob. (F-statistic) } & 0.000000 \\
\hline Durbin-Watson stat & 0.857077 & & & \\
\hline \multicolumn{5}{|c|}{ 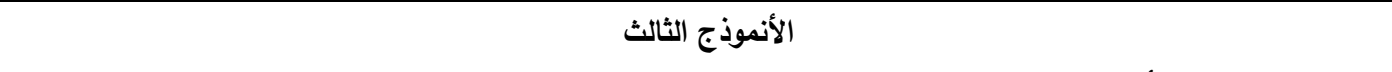 } \\
\hline \multicolumn{5}{|c|}{ أثر عجز الموازنة العامة والمتغيرات الضابطة في مؤشر التنمية المالية (SMC) } \\
\hline Variable & Coefficient & Std. Error & t-Statistic & Prob. \\
\hline BUD & 161.1897 & 50.19612 & 3.211198 & 0.0019 \\
\hline $\mathrm{C}$ & 33.88023 & 17.65443 & 1.919078 & 0.0587 \\
\hline \multicolumn{5}{|c|}{ Weighted Statistics } \\
\hline R-squared & 0.120641 & \multicolumn{2}{|c|}{ F-statistic } & 10.42655 \\
\hline Adjusted R-squared & 0.109070 & \multicolumn{2}{|c|}{ Prob. (F-statistic) } & 0.001835 \\
\hline Durbin-Watson stat & 0.572352 & & & \\
\hline \multirow{2}{*}{\multicolumn{5}{|c|}{ 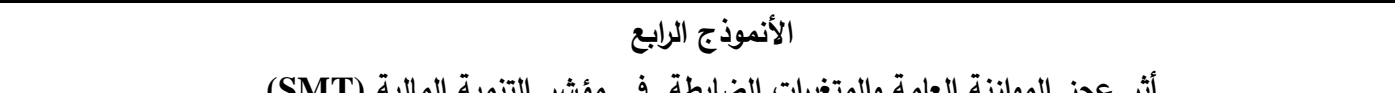 }} \\
\hline & & & & \\
\hline Variable & Coefficient & Std. Error & t-Statistic & Prob. \\
\hline BUD & 158.4789 & 36.88642 & 4.296401 & 0.0001 \\
\hline $\mathrm{C}$ & 41.30723 & 16.87979 & 2.447141 & 0.0167 \\
\hline \multicolumn{5}{|c|}{$\begin{array}{l}\text { Weighted Statistics } \\
\end{array}$} \\
\hline R-squared & 0.196052 & \multicolumn{2}{|c|}{ F-statistic } & 18.53348 \\
\hline Adjusted R-squared & 0.185474 & \multicolumn{2}{|c|}{ Prob. (F-statistic) } & 0.000049 \\
\hline Durbin-Watson stat & 0.836424 & & & \\
\hline
\end{tabular}

الجدول من عمل الباحثين بالاعتماد على مخرجات برنامج Eviews10

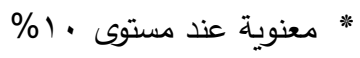

يمكن تشخيص ما يأتي:

• أكدت النماذج الأربعة على وجود تأثير معنوي لمتغير عجز الموازنة العامة (BUD) في مؤشرات

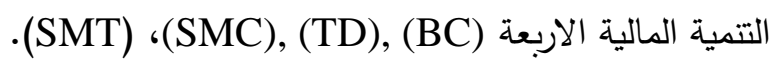

توافق تأثير متغير عجز الموازنة العامة (BUD) في مؤشري التمية المالية (BC)،)(TD) مع المنطق

الاقتصادي والدراسات التجريبية التي أطرت لهذه العلاقات، وبمعاملين بلغا (80.75528-)، -)

(72.26684 على التوالي، وهو ما جاء متوافقا مع الأطر النظرية والدراسات التجريبية التي اكدت

على أن انخفاض عجز الموازنة العامة يعمل على تعزيز التتمية المالية. • جاء الأثر الايجابي لمتغير عجز الموازنة العامة (BUD) في مؤشري التمية المالية (SMC)، مناقضاً للمنطق الاقتصادي، والذي يشير إلى زيادة مستويات عجز الموازنة العامة يعمل (SMT) على رفع مستويات تتمية القطاع المالي وبمعاملين بلغا (161.1897 )، (158.4789) على التوالي، وربما يعود ذلك إلى ان الادوات الحكومية من سندات واذونات خزانة والتي يطلق عليها عادة بالموجودات الامنة (Safe Asset) ونتيجة لتمتع اسواقها بالاستقرار النسبي وقدرتها على تسهيل عمل 
الوساطة المالية يمكن ان تساهم وبفاعلية في تطوير وتتمية الاسواق المالية وبالتالي القطاع المالي بدلاً Kutivadze (2011), Kumhof \& Tanner (2005). من عرقلتهان يظهر الأثر السلبي للمتغير الضابط النمو الاقتصادي (GRO) في مؤشري التتمية المالية (BC)

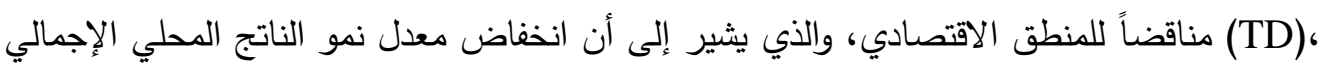
يعمل على رفع مستويات تتمية القطاع المالي وبمعاملي (1.233333-)، (1.337881-) على الى الثى التوالي، وربما يعود ذلك نتيجة الافتقار إلى سياسات الاقتصاد الكلي السليمة والتشريعات القانونية المؤاتية لجعل البيئة الاقتصادية داعمة للتأثير الايجابي لمعدل نمو الناتج المحلي الإجمالي على لتيات تتمية القطاع المالي. Huang(2006) (Benyah, 2010, 19) . استطاع متغير عجز الموازنة العامة (BUD) والمتغير الضابط (Control Variables) المرافق له النمو اقتصادي (GDP)، تقسير ما مقداره 51.4208\% \% 49.3570, من التغيرات في الحاصلة في مؤشري التتمية المالية (BD), (BC) على التوالي متخلياً للمتغيرات التوضيحية الاخرى غير الداخلة

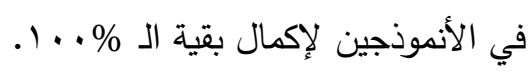

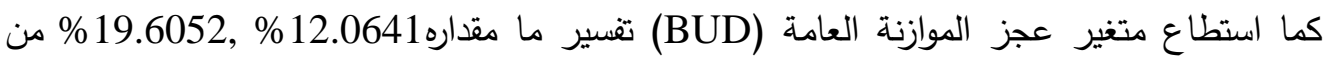
التغيرات في الحاصلة في مؤشري التتمية المالية (SMT), (SMC) على التوالي. فثل المتغير الضابط التضخم (INF) في إثبات تأثيره المعنوي في النماذج الاربعة لمؤشرات التنمية

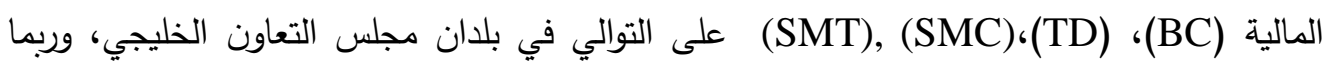

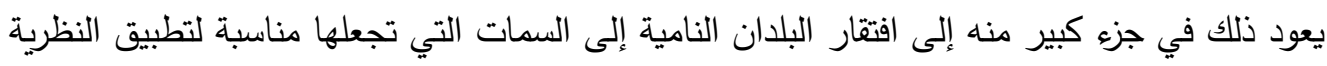
الاقتصادية السائدة، والناجم عن الطبيعة الخاصة لبيئتها السياسية والاقتصادية والاجتماعية (العادات والتقاليد) والدينية (كالمبادئ الدينية الإسلامية التي تؤكد على تجنب المحرمات وشبهاتها) لبلدان العينة

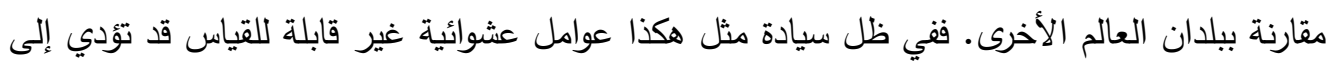

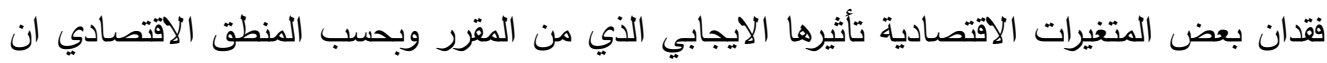
تمارسه في تتمية القطاع المالي. كما فثل متغير النمو الاقتصادي (GRO) في اثبات تأثيره المعنوي في مؤشري التتمية المالية للأنموذجين الثالث والرابع في بلدان مجلس التعاون الخليجي، وربما جاء ذلك نتيجة لفقدان العلاقة المباشرة بينه وبين تتمية القطاع المالي، فالمستويات المرتفعة منه يمكن ان تمارس تأثيرها في مؤشرات التتمية المالية من خلال تأثيرها في متغيرات أخرى تسهم في التأثير على تلى لتهن تتمية القطاع المالي مثل مستويات البنية التحتية الاقتصادية والمالية والمؤسساتية وغيرها.

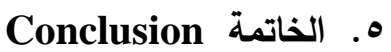

شكلت دراسة الآثار التي يمكن أن يتركها عجز الموازنة العامة في تتمية القطاع المالي الهدف الرئيس لهذا البحث، متخذاً من القطاع المالي بشقيه المصرفي والاسواق المالية في دول مجلس التعاون 


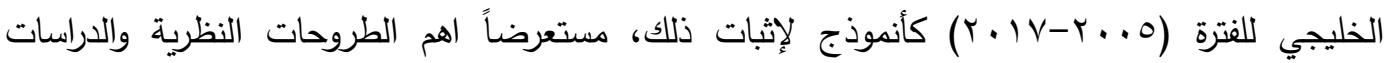
التجريبية التي تتاولت تلك الاثار، ومستخدماً أدوات القياس الاقتصادي الحديثة المتمثلة بمنهجية البيانات المزدوجة المتوازنة (Balanced Panel Data)، والتي اكدت نتائجها على تباين تأثير عجز الموازنة

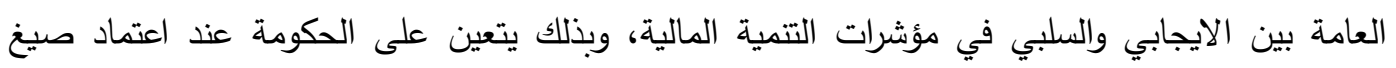
تمويل عجز الموازنة العامة مراعاة خصوصية أنموذجي القطاع المالي من حيث كونه قطاعاً مصرفياً أو لوائي

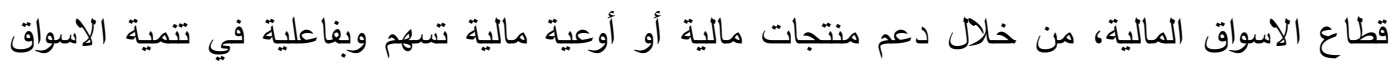
المالية، حيث تثير النتائج إلى أن استخدام الادوات الحكومية من سندات وأذونات خزانة والتي يطلق عليها

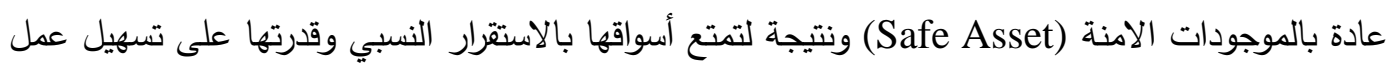

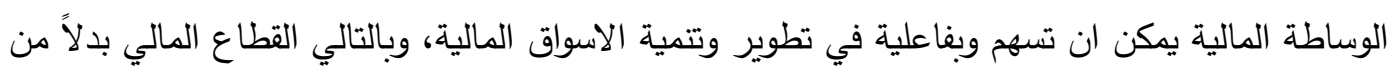

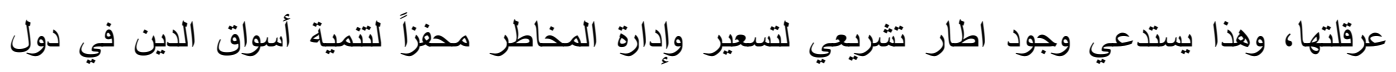

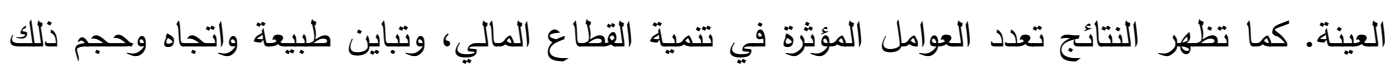
التأثير، وفي هذا الاتجاه مارس متغير النمو الاقتصادي دوراً سلبي التأثير في مؤشرات التتمية المالية،

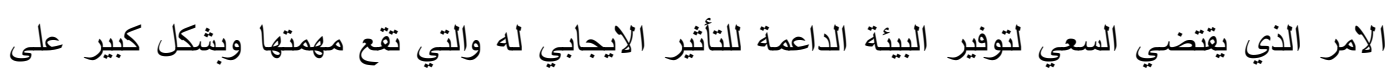

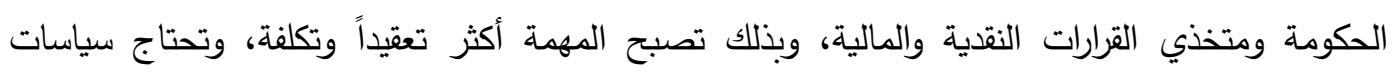
وبرامج طويلة الامد تقوم بتتفيذها الحكومة بدعم ومساعدة المنظمات الدولية في الجوانب الفنية والمالية. ومن جانب آخر، ينبغي إجراء المزيد من الدراسات والأبحاث حول العوامل الرئيسة المؤثرة في تتمية لأبهات

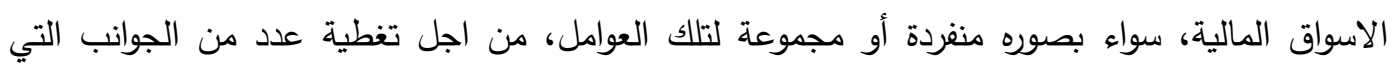
مازالت بحاجة لمزيد من الفحص والتدقيق ومنها السياسية، والقانونية، والمؤسساتية، والاجتماعية.

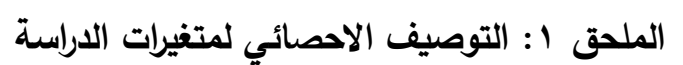

\begin{tabular}{|l|c|c|c|c|c|c|c|}
\hline & BC & BUD & GRO & INF & SMC & SMT & TD \\
\hline Mean & 56.72679 & 0.004600 & 4.854231 & 3.624359 & 34.62170 & 42.03623 & 53.07897 \\
\hline Median & 55.83000 & -0.014500 & 4.360000 & 2.900000 & 13.74441 & 25.95231 & 51.18000 \\
\hline Maximum & 105.1900 & 0.298000 & 26.17000 & 15.10000 & 331.2711 & 287.6240 & 100.0700 \\
\hline Minimum & 30.65000 & -0.213100 & -7.080000 & -4.900000 & 0.709515 & 0.856967 & 16.38000 \\
\hline Std. Dev. & 17.61857 & 0.098766 & 5.126712 & 3.569757 & 55.63128 & 50.53253 & 21.07920 \\
\hline Skewness & 0.528400 & 0.391658 & 1.325172 & 1.283942 & 3.446600 & 2.594597 & 0.214690 \\
\hline Kurtosis & 2.588478 & 3.341725 & 7.057323 & 4.961194 & 16.22363 & 11.10328 & 2.304043 \\
\hline & & & & & & & \\
\hline Jarque-Bera & 4.180075 & 2.373669 & 76.33014 & 33.93101 & 722.7369 & 300.9205 & 2.173351 \\
\hline Probability & 0.123683 & 0.305186 & 0.000000 & 0.000000 & 0.000000 & 0.000000 & 0.337336 \\
\hline & & & & & & & \\
\hline Sum & 4424.690 & 0.358800 & 378.6300 & 282.7000 & 2700.493 & 3278.826 & 4140.160 \\
\hline Sum Sq. Dev. & 23901.88 & 0.751114 & 2023.804 & 981.2237 & 238302.6 & 196622.3 & 34213.61 \\
\hline & & & & & & & \\
\hline Observations & 78 & 78 & 78 & 78 & 78 & 78 & 78 \\
\hline
\end{tabular}

الجدول من عمل الباحثين بالاعتماد على مخرجات برنامج Eviews10

TANMIYAT AL-RAFIDAIN (P-ISSN: 1609-591X; E-ISSN: 2664-276X) تنمية الرافدين 
أثر عجز الموازنة العامة في تعزيز تنمية القطاع المالي الدباغ والعراقي

الملحق ץ: الرسوم البيانية للبيانات المزدوجة لمتغيرات الدراسة 

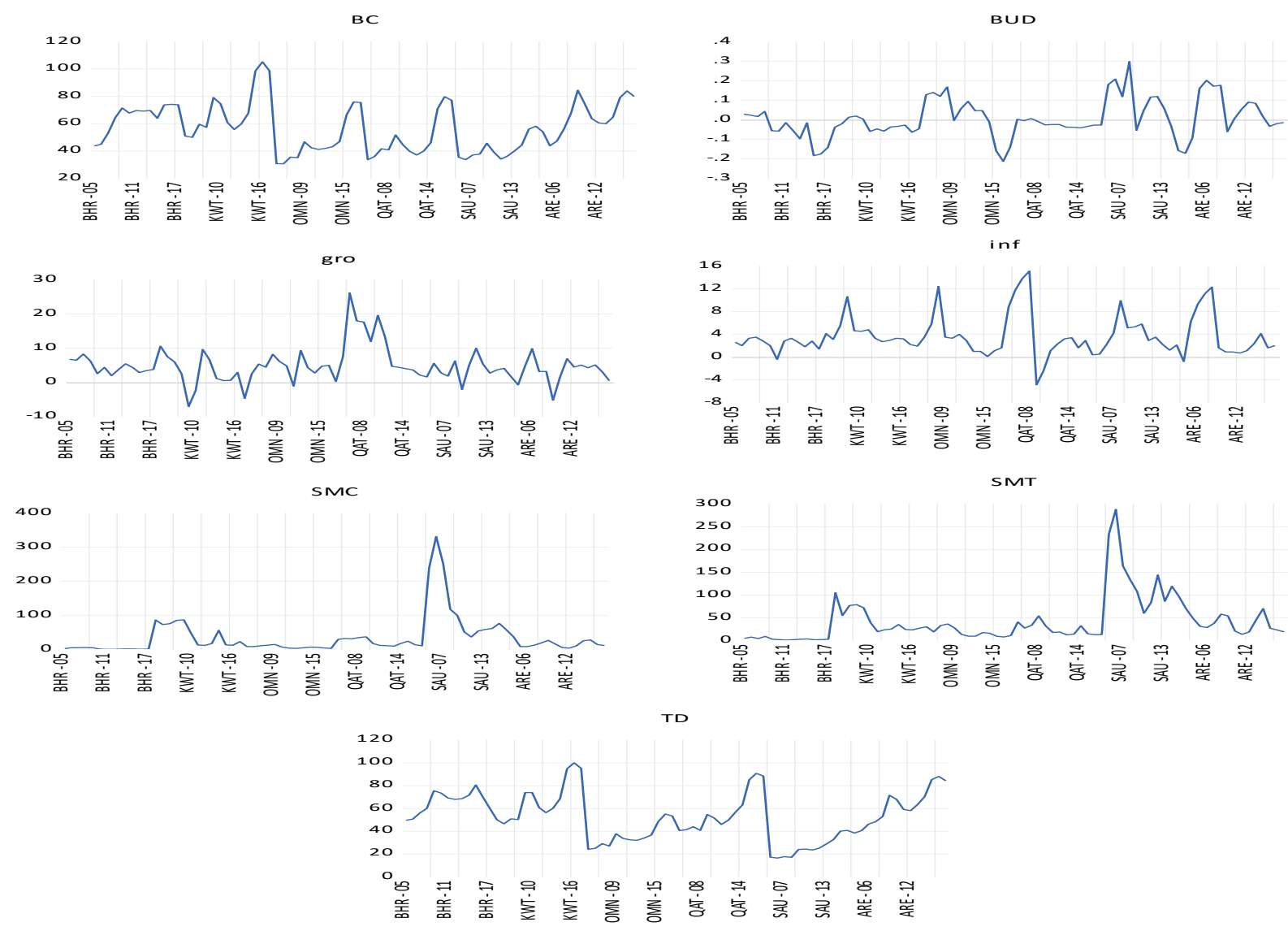

Eviews 9 الثكل من إعداد الباحثين بالاعتماد على نتائج برنامج

الملحق r: نتائج اختبار Levin-Lin-Chu,2002 لاستقرارية بيانات الـ Panel Data لمتغيرات

الاراسة بصيغتها الخطية

\begin{tabular}{|c|c|c|c|c|c|c|}
\hline \multirow{3}{*}{ Variables } & \multicolumn{3}{|c|}{ Level } & \multicolumn{3}{c|}{ First Difference } \\
\cline { 2 - 7 } & None & Intercept & $\begin{array}{c}\text { Trend and } \\
\text { Intercept }\end{array}$ & None & Intercept & $\begin{array}{c}\text { Trend and } \\
\text { Intercept }\end{array}$ \\
\hline BUD & -1.65527 & -197068 & -3.80474 & & & \\
Prob. & 0.0489 & 0.0244 & 0.0001 & & & \\
\hline GRO & -3.34875 & -4.53562 & -7.31091 & & & \\
Prob. & 0.0004 & 0.0000 & 0.0000 & & & \\
\hline INF & -2.95095 & -2.57692 & -4.02401 & & & \\
Prob. & 0.0016 & 0.0050 & 0.0000 & & & \\
\hline BC & 2.69380 & -5.62908 & -1.66411 & -5.75349 & -4.20746 & -1.95565 \\
Prob. & 0.9965 & 0.2646 & 0.0480 & 0.0000 & 0.0000 & 0.0253 \\
\hline SMC & -2.52617 & -5.85078 & -4.55139 & & & \\
Prob. & 0.0058 & 0.0000 & 0.0000 & & & \\
\hline
\end{tabular}

TANMIYAT AL-RAFIDAIN (P-ISSN: 1609-591X; E-ISSN: 2664-276X) تنمية الرافدين 


\begin{tabular}{|c|c|c|c|c|c|c|}
\hline \multirow{2}{*}{ Variables } & \multicolumn{3}{|c|}{ Level } & \multicolumn{3}{c|}{ First Difference } \\
\cline { 2 - 7 } & None & Intercept & $\begin{array}{c}\text { Trend and } \\
\text { Intercept }\end{array}$ & None & Intercept & $\begin{array}{c}\text { Trend and } \\
\text { Intercept }\end{array}$ \\
\hline SMT & -4.99317 & -3.81313 & -5.45476 & & & \\
Prob. & 0.0000 & 0.0001 & 0.0000 & & & \\
\hline TD & 2.80634 & 0.17560 & -2.92798 & -5.95292 & -5.08505 & -4.50441 \\
Prob. & 0.9975 & 0.5697 & 0.0017 & 0.0000 & 0.0000 & 0.0000 \\
\hline
\end{tabular}

• الجدول من عمل الباحث بالاعتماد على مخرجات برنامج 10 Eviews • - مالقيم في الجدول تمثل قيم Statistict.

• تم اعتماد فترة الابطاء بالاستتاد إلى Automatic selection of maximum lags ضمن برنامج Eviews

\section{Refrence}

AL-Omary, Muhammad Walid, Al-Hayt, Nahil Ismail Saqf, 2016, The Impact of Technical Efficiency and Exports on the Profitability of the Jordanian Pharmaceutical Industry Enterprises, The Jordanian Journal of Business Administration, (12) 4, 877-898.

Al-Iraqi, Bashar, 2018, Factors Determining the Revenues of Monetary Issue in the Arab Countries, Journal of Development and Economic Policies, (20) 1, 71-104.

Al-Iraqi, Bashar, 2017, The Impact of Money Supply Fluctuations on Stock Prices: Developing a Model for Financial Markets in the Gulf Cooperation Council Countries, (24) 3, 445-474, Arab Journal of Management Sciences.

Ismihan, Mustafa, \& Ozkan, F. Gulcin (2010). Public Debt and Financial Development: A Theoretical Exploration., Discussion Papers in Economics No.14, University of York, Heslington.

Hauner, David, 2009. Public debt and financial development. Journal of Development Economics 88 (1), 171-183

Caballero , Ricardo J. \& Krishnamurthy, Arvind Krishnamurthy, 2004, Fiscal policy and financial depth. NBER Working Paper, No. 10532.

Christensen, JAKOB, 2005. Domestic debt markets in Sub-Saharan Africa. IMF Staff Papers 52 (3), 518-538.

Ali, Amjad , Ahmad, Farooq and Ur- Rahman, Fazal, 2016, Impact of Government Borrowing on Financial Development (A case study of Pakistan), MPRA Paper No. 78100.

Hussain , Adnan, Mohammad ,Sulaiman D., Akram ,Kamran\& Lal, Irfan, 2009, Effectiveness of Government Expenditure Crowding-In or Crowding-Out: Empirical Evidence in Case of Pakistan, European Journal of Economics, Finance And Administrative Sciences - Issue 16,124-149.

Rehman, Hafeez-ur, Khan, Sajawal, and Khan, M. Arshad (2009). What determines private investment? The case of Pakistan. South Asian Studies A Research Journal of South Asian Studies, 24(1), 52-68.

TANMIYAT AL-RAFIDAIN (P-ISSN: 1609-591X; E-ISSN: 2664-276X) تنمية الرافدين 
Ardagna, Silvia, Caselli, Francesco, and Lane, Timothy, (2007). Fiscal discipline and the cost of public debt service: some estimates for OECD countries., European Central Bank, WORKING PAPER SERIES NO. 411

Naqvi, Naveed, H. (2002). Crowding-in or Crowding-out? Modelling the relationship between public and private fixed capital formation using cointegration analysis: the case of Pakistan 1964-2000. The Pakistan Development Review, 255-275.

Takyi Paul Owusu \& Obeng, Camara Kwesi, 2013, Determinants of financial development in Ghana, International Journal of Development and Sustainability, Volume 2 Number 4,1-13.

Alenoghena ,Raymond, 2018, Financial Market Development and Fiscal Deficit Financing In Nigeria, the Biennial Conference of the Economic Society of South Africa: University of Cape Town.

Hauner, David (2009). Public Debt and Financial Development. Journal of Development Economics, 88, 171-183.

Shetta, Samah \& Kamaly Ahmed (2014), Does the Budget Deficit Crowd-Out Private Credit From the Banking Sector? The Case of Egypt, Topics in Middle E astern and African Economies,16(2):251-279. Ayadi ,Rym , de Groen, Willem Pieter, Chmelar Ales \& Pyykkö, Elina, 2013, Banking and Insurance in the GCC Countries: Is there Regulatory Convergence with the EU?,NR.4.

Ismihan, Mustafa, \& Ozkan, F. Gulcin (2010). Public Debt and Financial Development: A Theoretical Exploration., Discussion Papers in Economics No.14, University of York, Heslington.

Janda, Karel \& Kravtsov, Oleg, 2017, Time-varying Effects of Public Debt on the Financial and Banking Development in the Central and Eastern Europe, MPRA Paper No. 77325.

İlgün ,Miraç Fatih, 2016, Financial Development and Domestic Public Debt in Emerging Economies: A Panel Cointegration Analysis, Journal of Applied Economics and Business Research JAEBR, 6(4): 284-296 .

Andrés,Javier, Arce, "scar\& Thomas ,Carlos ,2016, When Fiscal Consolidation Meets Private Deleveraging, Social Science Research Network, Rochester, NY.

Kutivadze ,Natia,2011,Public Debt and Financial Development, Working Paper n. 2011-13

Svirydzenka ,Katsiaryna,2016,Strategy, Policy, and Review Department Introducing a New Broad-based Index of Financial Development, International Monetary Fund WP/16/5 IMF Working Paper

Gupta, Gaurav \& Mahakud, Jitendram,2019, Alternative measure of financial development and investment-cash flow sensitivity: evidence from an emerging Economy, Financial Innovation, vol. 5, Article No. 1:P.9

Čihák, Martin et al.,2012, Financial Development in 205 Economies, 1960 to 2010, NBER Working Paper No. 18946, Cambridge.

TANMIYAT AL-RAFIDAIN (P-ISSN: 1609-591X; E-ISSN: 2664-276X) تنمية الرافدين 
Asiama, Johnson P\& Mobolaji, Hakeem,2011, Trade and Financial Openness, Institutional Quality, and Financial Development in Sub-Sahara Africa (SSA), Nigeria.

Aluko, Olufemi A. \& Ajayi, Michael Adebayo 2018,Determinants of banking sector development: Evidence from Sub-Saharan African countries, Borsa - Istanbul Review , 18(2),122-139

$\mathrm{Xu}$, Zhenhui, 2000, Financial development, investment, and economic growth, Economic Inpoury,38(2).331-344.

Demetriades, Panicos O. \& Hussein, Khaled A. ,1996, Does financial development cause economic growth? Time-series evidence from 16 countries, Journal of Development Economics.51(2): 387-411

Ardicad, Oya Pinar \& Damar, H. Evren,2006, Financial Sector Deepening and Economic Growth: Evidence From Turkey, MPRA Paper 4077,University Library of Munich, Germany.

Ghali, Khalifa H. ,1999, Financial Development and Economic Growth: The Tunisian Experience, Review of Development Economics,3(3): 310-322.

Khan ,Mohsin S. \& Senhadji ,Abdelhak S. ,2003, Financial Development and Economic Growth: A Review and New Evidence, Journal of African Economies, (12)2, 89-110

Gregorio, Jose De \& Guidotti, Pablo E.,1995, Financial development and economic growth, World Development, 23(3):433-448.

Levine, Ross \& Zervos, Sara (1998). Stock Markets, Banks, and Growth. American Economic Review, 88(3):537-558

Nazir ,Mian Sajid , Nawaz ,Muhammad Musarat \& Gilani ,Usman Javed ,2010, Relationship between economic growth and stock market development, African Journal of Business Management Vol. 4(16), pp. 3473-3479.

Arestis,Philip \& Demetriades, Panicos,1997, Financial Development and Economic Growth: Assessing the Evidence, Wiley, The Economic Journal, Vol. 107, No. 442 (May, 1997), pp. 783-799.

Demirguc-Kunt , Asli \& Levine, Ross,1996, Stock market development and financial intermediaries: stylized facts, World Bank Economic Review, 10(2): 291-321.

Ake ,Boubakari Ake\& Ognaligui ,Rachelle Wouono, 2010, Financial Stock Market and Economic Growth in Developing Countries: The Case of Douala Stock Exchange in Cameroon, International Journal of Business and Management Vol. 5, No. 5,82-88.

Balogun ,Wakilat Olabisi , Asso ,Jauhari. B.\& Hassan,Sallahuddin B. ,2016, Long Term Effect of Liquidity on Stock Market Development,Journal of Economics and Sustainable Development Vol.7, No.4.

Zang, Hyoungsoo \& Kim, Young Chul (2007). Does financial development precede growth? Robinson and Lucas might be right. Applied Economics Letters, 14(1), 15-19

TANMIYAT AL-RAFIDAIN (P-ISSN: 1609-591X; E-ISSN: 2664-276X) تنمية الرافدين 
Baltagi, Badi H., Demetriades, Panicos O., \& Law, Siong H. (2009). Financial development and openness: Evidence from panel data. Journal of Development Economics, 89(2), 285-296

Law, Siong Hook \& Habibullah, Muzafar Shah (2009). The determinants of financial development: Institutions, openness and financial liberalisation. South African Journal of Economics, 77(1), 45-58

Odhiambo ,Nicholas M ,2012, The Impact Of Inflation On Financial Sector Development: Experience From Zambia, Journal of Applied Business Research 28(6):1497-1508

Gozgor, Giray (2015). Causal relation between economic growth and domestic credit in the economic globalization: Evidence from the Hatemi-J's test. The Journal of International Trade and Economic Development, 24(3), 395-408.

Peia, Oana \& Roszbach, Kasper (2015). Finance and growth: time series evidence on causality. Journal of Financial Stability, 19, 105-118.

Boyd,John H., Levine, Ross\& Smith,Bruce D.,2001, The impact of inflation on financial sector performance, Journal of Monetary Economics, Volume 47, Issue 2, Pages 221-248.

Bittencourt, Manoel,2008, Inflation and Financial Development: Evidence from Brazil, United Nation University -WIDER, Research Paper No.14

Ben Naceur ,Sami, Cherif, Mondher \& Kandil, Magda ,2014 What drives the development of the MENA financial sector?,Borsa _Istanbul Review 144,212-223.

Gujarati, Damodar N., 2004, Basic Econometrics, Fourth Edition, The McGraw-Hill Companies

Torres-Reyna, Oscar, 2007, Panel Data Analysis Fixed and Random Effects using Stata (v. 4.2)

Gil-García, J. Ramón \& Puron-Cid, y Gabriel Puron-Cid, 2013, Using Panel Data Techniques for Social Science Research: an Illustrative Case and Some Guidelines, C I E N C I A e r g o -s um , (21)3, 203-216.

Clark, Paul, Crawford, Claire, Steele, Fiona \& Vignoles, Anna, 2010, The Choice Between Fixed and Random Effects Models: Some Considerations For Educational Research, Centre for Market and Public Organisation Bristol Institute of Public Affairs University of Bristol, Working Paper No. $10 / 240$. 